SUPPLEMENTARY CONTENT

\title{
SYNTHESIS OF NOVEL DISPIRO 1,4-BENZOTHIAZINE HYBRID HETEROCYCLES THROUGH 1,3-DIPOLAR CYCLOADDITION
}

Karuppiah Malathi, Veerappan Jeyachandran, Karumpan Kalaiselvan and Raju Ranjith Kumar*

Department of Organic Chemistry, School of Chemistry, Madurai Kamaraj University Madurai 625021, Tamil Nadu, India

\begin{tabular}{|c|c|}
\hline Table of Content & Page No \\
\hline Experimental - General & S3 \\
\hline Data for all compounds & S3 \\
\hline${ }^{1}$ H NMR spectra of compound 9a & S12 \\
\hline${ }^{13} \mathrm{C}$ NMR spectra of compound $9 \mathrm{a}$ & $\mathrm{S} 12$ \\
\hline${ }^{1}$ H NMR spectra of compound $9 \mathbf{b}$ & $\mathrm{S} 13$ \\
\hline${ }^{13} \mathrm{C}$ NMR spectra of compound $\mathbf{9 b}$ & $\mathrm{S} 13$ \\
\hline${ }^{1} \mathrm{H}$ NMR spectra of compound $\mathbf{1 0 a}$ & S14 \\
\hline${ }^{13} \mathrm{C}$ NMR spectra of compound 10a & S14 \\
\hline${ }^{1}$ H NMR spectra of compound $\mathbf{1 0 b}$ & $\mathrm{S} 15$ \\
\hline${ }^{13} \mathrm{C}$ NMR spectra of compound $\mathbf{1 0 b}$ & S15 \\
\hline${ }^{1} \mathrm{H}$ NMR spectra of compound 11a & S16 \\
\hline${ }^{13} \mathrm{C}$ NMR spectra of compound 11a & S16 \\
\hline${ }^{1}$ H NMR spectra of compound 11b & S17 \\
\hline${ }^{13} \mathrm{C}$ NMR spectra of compound $\mathbf{1 1 b}$ & $\mathrm{S} 17$ \\
\hline${ }^{1} \mathrm{H}$ NMR spectra of compound $\mathbf{1 2 a}$ & $\mathrm{S} 18$ \\
\hline${ }^{13} \mathrm{C}$ NMR spectra of compound 12a & S18 \\
\hline${ }^{1} \mathrm{H}$ NMR spectra of compound $\mathbf{1 2 b}$ & S19 \\
\hline${ }^{13} \mathrm{C}$ NMR spectra of compound $\mathbf{1 2 b}$ & S19 \\
\hline DEPT-135 NMR spectra of compound $\mathbf{1 2 b}$ & $\mathrm{S} 20$ \\
\hline H,H COSY spectra of compound12b & $\mathrm{S} 20$ \\
\hline C,H COSY spectra of compound $\mathbf{1 2 b}$ & S21 \\
\hline HMBC spectra of compound $\mathbf{1 2 b}$ & S21 \\
\hline${ }^{1}$ H NMR spectra of compound 14a & S22 \\
\hline${ }^{13} \mathrm{C}$ NMR spectra of compound $\mathbf{1 4 a}$ & S22 \\
\hline${ }^{1} \mathrm{H}$ NMR spectra of compound $\mathbf{1 4 b}$ & S23 \\
\hline${ }^{13} \mathrm{C}$ NMR spectra of compound $\mathbf{1 4 b}$ & S23 \\
\hline${ }^{1} \mathrm{H}$ NMR spectra of compound $\mathbf{1 4 c}$ & S24 \\
\hline${ }^{13} \mathrm{C}$ NMR spectra of compound $\mathbf{1 4 c}$ & S24 \\
\hline${ }^{1} \mathrm{H}$ NMR spectra of compound $\mathbf{1 4 d}$ & S25 \\
\hline${ }^{13} \mathrm{C}$ NMR spectra of compound 14d & S25 \\
\hline${ }^{1} \mathrm{H}$ NMR spectra of compound $\mathbf{1 5 a}$ & S26 \\
\hline${ }^{13} \mathrm{C}$ NMR spectra of compound $\mathbf{1 5 a}$ & S26 \\
\hline${ }^{1} \mathrm{H}$ NMR spectra of compound $\mathbf{1 5 b}$ & S27 \\
\hline${ }^{13} \mathrm{C}$ NMR spectra of compound $\mathbf{1 5 b}$ & $\mathrm{S} 27$ \\
\hline
\end{tabular}




\begin{tabular}{|l|l|}
\hline${ }^{1}$ H NMR spectra of compound 15c & S28 \\
\hline${ }^{13}$ C NMR spectra of compound 15c & S28 \\
\hline${ }^{1}$ H NMR spectra of compound 15d & S29 \\
\hline${ }^{13}$ C NMR spectra of compound 15d & S29 \\
\hline${ }^{1}$ H NMR spectra of compound 16a & S30 \\
\hline${ }^{13}$ C NMR spectra of compound 16a & S30 \\
\hline${ }^{1}$ H NMR spectra of compound 16b & S31 \\
\hline${ }^{13}$ C NMR spectra of compound 16b & S31 \\
\hline${ }^{1}$ H NMR spectra of compound 16c & S32 \\
\hline${ }^{13}$ C NMR spectra of compound 16c & S32 \\
\hline${ }^{1}$ H NMR spectra of compound 16d & S33 \\
\hline${ }^{13}$ C NMR spectra of compound 16d & S33 \\
\hline
\end{tabular}




\section{EXPERIMENTAL}

General. The melting points were measured in open capillary tubes and are uncorrected. The ${ }^{1} \mathrm{H},{ }^{13} \mathrm{C}$ and the 2D NMR spectra were recorded on a Bruker (Avance) $300 \mathrm{MHz} \mathrm{NMR}$ instrument using TMS as internal standard and $\mathrm{CDCl}_{3}$ or $\mathrm{DMSO}-\mathrm{d}_{6}$ as solvent. Standard Bruker software was used throughout. Chemical shifts are given in parts per million ( $\delta$-scale) and the coupling constants are given in Hertz. Elemental analyses were performed on a Perkin Elmer 2400 Series II Elemental CHNS analyzer. Silica gel-G plates (Merck) were used for tlc analysis with a mixture of petroleum ether $\left(60-80^{\circ} \mathrm{C}\right)$ and ethyl acetate as eluent. All the chemicals were purchased from Sigma-Aldrich and used without any further purification.

General procedure for the synthesis of dispiro 1,4-benzothiazine hybrid heterocycles: A mixture of $(3,1 \mathrm{mmol})$, acenaphthylene-1,2-dione or isatins $(4$ or $\mathbf{1 3}, 1.2 \mathrm{mmol})$ and the respective $\alpha$-amino acid $(\mathbf{5}-\mathbf{8}, 1.5 \mathrm{mmol})$ in ethanol $(10 \mathrm{~mL})$ was heated to reflux on a water bath for $3 \mathrm{~h}$. After completion of the reaction as evident from TLC, the mixture was poured into crushed ice and the resulting solid was filtered off and purified by column chromatography on silica gel employing ethyl acetate/petroleum ether $(10: 90 \mathrm{v} / \mathrm{v})$ as eluent to obtain pure products 9-12 and 14-16.

Methyl 1'-methyl-[spiro[2',2"]-acenapthene-1"'-one]-3-oxo-3,4-dihydrospiro[benzo[b][1,4] thiazine-2,3'-pyrrolidine]-4'-carboxylate (9a): Obtained as colourless solid; Yield 75\%; m.p. 219-221 ${ }^{\circ} \mathrm{C}$; Anal. Calcd. for $\mathrm{C}_{25} \mathrm{H}_{20} \mathrm{~N}_{2} \mathrm{O}_{4} \mathrm{~S}$ : C, 67.55; H, 4.54; N, 6.30; S, 7.21. Found: C, 67.62; H, 4.46; N, 6.23; S, 7.28. ${ }^{1} \mathrm{H}$ NMR (300 MHz, $\left.\mathrm{CDCl}_{3}\right) \delta_{\mathrm{H}}: 2.08(\mathrm{~s}, 3 \mathrm{H}), 3.49(\mathrm{t}$, $J=8.4 \mathrm{~Hz}, 1 \mathrm{H}), 3.73-3.82(\mathrm{~m}, 3 \mathrm{H}), 4.19(\mathrm{t}, J=9.3 \mathrm{~Hz}, 1 \mathrm{H}), 4.94(\mathrm{t}, J=9.0 \mathrm{~Hz}, 1 \mathrm{H}), 5.92(\mathrm{~d}$, $J=7.8 \mathrm{~Hz}, 1 \mathrm{H}), 6.34(\mathrm{t}, J=7.5 \mathrm{~Hz}, 1 \mathrm{H}), 6.44(\mathrm{t}, J=7.8 \mathrm{~Hz}, 1 \mathrm{H}), 6.66(\mathrm{~d}, J=7.5 \mathrm{~Hz}, 1 \mathrm{H}), 7.41$ (d, $J=4.5 \mathrm{~Hz}, 1 \mathrm{H}), 7.44-7.50(\mathrm{~m}, 2 \mathrm{H}), 7.52-7.56(\mathrm{~m}, 2 \mathrm{H}), 7.63(\mathrm{~d}, J=7.8 \mathrm{~Hz}, 1 \mathrm{H}), 7.78(\mathrm{~d}, J=8.1$

$\mathrm{Hz}, 1 \mathrm{H}), 9.57(\mathrm{~s}, 1 \mathrm{H}) .{ }^{13} \mathrm{C} \mathrm{NMR}(75 \mathrm{MHz}, \mathrm{CDCl} 3) \delta_{\mathrm{C}}: 34.8,39.3,39.6,39.8,46.6,51.6$, 
$54.1,58.1,81.2,114.9,119.4,121.2,122.3,125.1,126.5,126.6,127.1,127.7,129.1,130.3$, $131.3,135.6,165.6,170.1,202.8$.

Ethyl 1'-methyl-[spiro[2',2"]-acenapthene-1"-one]-3-oxo-3,4-dihydrospiro[benzo[b] [1,4] thiazine-2,3'-pyrrolidine]-4'-carboxylate (9b): Obtained as colourless solid; Yield 72\%; m.p. 176-178 ${ }^{\circ}$; Anal. Calcd. for $\mathrm{C}_{26} \mathrm{H}_{22} \mathrm{~N}_{2} \mathrm{O}_{4} \mathrm{~S}$ : C, 68.10; H, 4.84; N, 6.11; S, 6.99. Found: C, 68.18; H, 4.77; N, 6.03; S, 7.07. ${ }^{1} \mathrm{H}$ NMR $\left(300 \mathrm{MHz} \mathrm{CDCl}_{3}\right) \delta_{\mathrm{H}}: 1.33(\mathrm{t}, J=7.2 \mathrm{~Hz}, 3 \mathrm{H}), 2.08$ $(\mathrm{s}, 3 \mathrm{H}), 3.51(\mathrm{t}, J=8.4 \mathrm{~Hz}, 1 \mathrm{H}), 4.19(\mathrm{t}, J=9.3 \mathrm{~Hz}, 1 \mathrm{H}), 4.29(\mathrm{dd}, J=13.8,6.6 \mathrm{~Hz}, 2 \mathrm{H}), 4.88(\mathrm{t}$, $J=9.0 \mathrm{~Hz}, 1 \mathrm{H}), 5.87(\mathrm{~d}, J=7.5 \mathrm{~Hz}, 1 \mathrm{H}), 6.39(\mathrm{t}, J=7.5 \mathrm{~Hz}, 1 \mathrm{H}), 6.50(\mathrm{t}, J=6.6 \mathrm{~Hz}, 1 \mathrm{H}), 6.71(\mathrm{~d}$, $J=7.5 \mathrm{~Hz}, 1 \mathrm{H}), 7.42-7.52(\mathrm{~m}, 3 \mathrm{H}), 7.56-7.62(\mathrm{~m}, 2 \mathrm{H}), 7.77(\mathrm{~d}, J=7.6 \mathrm{~Hz}, 1 \mathrm{H}), 8.44(\mathrm{~s}, 1 \mathrm{H})$. ${ }^{13} \mathrm{C}$ NMR $\left(75 \mathrm{MHz}, \mathrm{CDCl}_{3}\right) \delta_{\mathrm{C}}: 14.2,35.2,47.1,54.6,58.6,61.2,81.6,115.1,117.5,119.9$, $121.4,123.1,125.4,127.0,127.1,127.5,128.1,129.5,130.6,131.7,135.5,136.0,142.1$, 166.7, 169.9, 203.1.

Methyl [spiro[3',2"]-acenapthene-1"-one]-3-oxo-5'-phenyl-3,4-dihydrospiro[benzo[b][1,4] thiazine-2,3'-pyrrolidine]-4'-carboxylate (10a): Obtained as pale white solid; Yield 68\%; m.p. 204-206 ${ }^{\circ}$; Anal. Calcd. for $\mathrm{C}_{31} \mathrm{H}_{24} \mathrm{~N}_{2} \mathrm{O}_{4} \mathrm{~S}$ : C, 71.13; H, 4.38; N, 5.53; S, 6.33. Found: C, 71.21; H, 4.32; N, 5.46; S, $6.41{ }^{1} \mathrm{H}$ NMR (300 MHz, DMSO-D $\left.6 / \mathrm{CDCl}_{3}\right) \quad \delta_{\mathrm{H}}: 1.20(\mathrm{t}$, $J=6.6 \mathrm{~Hz}, 3 \mathrm{H}), 4.13(\mathrm{~d}, J=6.9 \mathrm{~Hz}, 2 \mathrm{H}), 4.79$ (d, $J=10.5 \mathrm{~Hz}, 1 \mathrm{H}), 5.60$ (d, $J=6.3 \mathrm{~Hz}, 1 \mathrm{H}), 5.83$ (d, $J=7.8 \mathrm{~Hz}, 1 \mathrm{H}), 6.54(\mathrm{~d}, J=7.8 \mathrm{~Hz}, 1 \mathrm{H}), 6.58-6.63(\mathrm{~m}, 1 \mathrm{H}), 6.93(\mathrm{~d}, \mathrm{~J}=7.5 \mathrm{~Hz}, 1 \mathrm{H}), 7.30-7.46$ $(\mathrm{m}, 5 \mathrm{H}), 7.56-7.60(\mathrm{~m}, 2 \mathrm{H}), 7.62-7.75(\mathrm{~m}, 4 \mathrm{H}), 7.86(\mathrm{~d}, J=7.8 \mathrm{~Hz}, 1 \mathrm{H}), 9.83(\mathrm{~s}, 1 \mathrm{H}) .{ }^{13} \mathrm{C}$ NMR (75 MHz, CDCl3) $\delta_{\mathrm{C}}: 13.1,56.3,59.1,59.9,61.7,76.6,114.5,115.7,119.0,120.5$, $121.7,124.2,126.3,126.5,126.6,126.8,127.0,127.3,128.3,129.7,130.2,135.9,137.3$, $139.1,139.9,163.9,168.2,202.4$

Ethyl [spiro[3',2"]-acenapthene-1"-one]-3-oxo-5'-phenyl-3,4-dihydrospiro[benzo[b][1,4] thiazine-2,3'-pyrrolidine]-4'-carboxylate (10b): Obtained as pale white solid; Yield 86\%; m.p. $201-203^{\circ} \mathrm{C}$; Anal. Calcd. for $\mathrm{C}_{31} \mathrm{H}_{24} \mathrm{~N}_{2} \mathrm{O}_{4} \mathrm{~S}$ : C, 71.52; H, 4.65; N, 5.38; O, 12.29; S, 6.16 
C, 71.44; H, 4.71; N, 5.45; S, 6.10. ${ }^{1} \mathrm{H}$ NMR (300 MHz, DMSO-D $\left.6 / \mathrm{CDCl}_{3}\right) \quad \delta_{\mathrm{H}}: 1.20(\mathrm{t}$, $J=6.9 \mathrm{~Hz}, 3 \mathrm{H}), 4.17(\mathrm{dd}, J=14.1,6.9 \mathrm{~Hz}, 1 \mathrm{H}), 4.76(\mathrm{~d}, J=10.2 \mathrm{~Hz}, 1 \mathrm{H}), 5.64-5.69(\mathrm{~m}, 2 \mathrm{H}), 6.57$ (t, $J=7.8 \mathrm{~Hz}, 1 \mathrm{H}), 6.67(\mathrm{t}, J=7.5 \mathrm{~Hz}, 1 \mathrm{H}), 7.02(\mathrm{~d}, J=7.8 \mathrm{~Hz}, 1 \mathrm{H}), 7.30-7.41(\mathrm{~m}, 5 \mathrm{H}), 7.57(\mathrm{~d}$, $J=7.8 \mathrm{~Hz}, 1 \mathrm{H}), 7.65(\mathrm{~d}, J=6.9 \mathrm{~Hz}, 1 \mathrm{H}), 7.68-7.71(\mathrm{~m}, 3 \mathrm{H}), 7.93(\mathrm{dd}, J=6.3,2.4 \mathrm{~Hz}, 1 \mathrm{H}) .{ }^{13} \mathrm{C}$ NMR (75 MHz, CDCl3) $\delta_{\mathrm{C}}: 14.1,22.6,29.6,57.2,60.2,61.2,62.8,77.9,115.3,117.5$, $120.3,121.5,123.5,127.5,127.6,127.8,128.0,128.2,128.4,128.6,129.4,130.8,131.1$, $136.1,136.1,137.9,140.3,140.8,165.8,169.1,203.6$.

Methyl [spiro[3',2"]-acenapthene-1"'-one]-3-oxo-1',3,3',4,5',6',7',7a'-octahydrospiro[benzo [b][1,4]thiazine-2,2'-pyrrolizine]-1'-carboxylate (11a): Obtained as pale white solid; Yield 75\%; m.p. $199-201^{\circ} \mathrm{C}$; Anal. Calcd. for $\mathrm{C}_{27} \mathrm{H}_{22} \mathrm{~N}_{2} \mathrm{O}_{3} \mathrm{~S}: \mathrm{C}, 68.92 ; \mathrm{H}, 4.71 ; \mathrm{N}, 5.95 ; \mathrm{S}, 6.81$ Found: C, C, 68.99; H, 4.64; N, 6.03; S, 6.87. ${ }^{1} \mathrm{H}$ NMR $\left(300 \mathrm{MHz}, \mathrm{DMSO}-\mathrm{D}_{6} / \mathrm{CDCl}_{3}\right) \quad \delta_{\mathrm{H}}$ : $1.11(\mathrm{t}, J=7.2 \mathrm{~Hz}, 3 \mathrm{H}), 2.17(\mathrm{~d}, J=5.7 \mathrm{~Hz}, 3 \mathrm{H}), 2.42-2.45(\mathrm{~m}, 1 \mathrm{H}), 2.65(\mathrm{t}, J=4.8 \mathrm{~Hz}, 1 \mathrm{H})$, 3.72-3.78 (m, 1H), 3.94-4.03 (m, 1H), $4.45(\mathrm{dd}, J=16.8 \mathrm{~Hz}, 7.8 \mathrm{~Hz}, 1 \mathrm{H}), 4.83(\mathrm{~d}, J=9.6 \mathrm{~Hz}$, $1 \mathrm{H} 0,5.30(\mathrm{~s}, 1 \mathrm{H}), 6.03(\mathrm{~d}, J=7.5 \mathrm{~Hz}, 1 \mathrm{H}), 6.80(\mathrm{t}, J=7.5 \mathrm{~Hz}, 1 \mathrm{H}), 6.88(\mathrm{t}, J=7.5 \mathrm{~Hz}, 1 \mathrm{H}), 7.19$ (d, $J=6.0 \mathrm{~Hz}, 1 \mathrm{H}), 7.64(\mathrm{~d}, J=8.7 \mathrm{~Hz}, 2 \mathrm{H}), 7.79$ (d, $J=6.9 \mathrm{~Hz}, 1 \mathrm{H}), 7.82(\mathrm{~d}, \mathrm{~J}=6.9 \mathrm{~Hz}, 1 \mathrm{H}), 7.95$ $(\mathrm{d}, \mathrm{J}=8.4 \mathrm{~Hz}, 1 \mathrm{H}) .{ }^{13} \mathrm{C} \mathrm{NMR}(75 \mathrm{MHz}, \mathrm{CDCl} 3) \delta_{\mathrm{C}}: 29.2,30.7,39.2,39.4,40.0,40.2,46.1$, $51.4,52.7,64.3,66.2,81.8,115.7,117.8,119.4,122.9,124.6,124.6,124.8,125.8,127.2$, $129.6,130.6,132.1,134.4,162.1,169.5,205.3$

Ethyl [spiro[3',2"]-acenapthene-1"'-one]-3-oxo-1',3,3',4,5',6',7',7a'-octahydrospiro[benzo[b] [1,4]thiazine-2,2'-pyrrolizine]-1'-carboxylate (11b): Obtained as pale white solid; Yield 84\%; m.p. $197-199^{\circ} \mathrm{C}$; Anal. Calcd. for $\mathrm{C}_{28} \mathrm{H}_{24} \mathrm{~N}_{2} \mathrm{O}_{4} \mathrm{~S}$ : C, 69.40; H, 4.99; N, 5.78; S, 6.62. Found: C, 69.33; H, 5.86; N, 5.72; S, 6.69. ${ }^{1} \mathrm{H}$ NMR (300 MHz, DMSO-D $\left.6 / \mathrm{CDCl}_{3}\right) \delta_{\mathrm{H}}: 1.11(\mathrm{t}$, $J=7.2 \mathrm{~Hz}, 3 \mathrm{H}), 2.17(\mathrm{~d}, J=5.7 \mathrm{~Hz}, 3 \mathrm{H}), 2.42-2.45(\mathrm{~m}, 1 \mathrm{H}), 2.65(\mathrm{t}, J=4.8 \mathrm{~Hz}, 1 \mathrm{H}), 3.72-3.78$ (m, 1H), 3.94-4.03 (m, 1H), 4.45 (dd, $J=16.8,7.8 \mathrm{~Hz}, 1 \mathrm{H}), 4.83$ (d, J=9.6Hz, 1H), 5.30 (s, $1 \mathrm{H}), 6.03(\mathrm{~d}, J=7.5 \mathrm{~Hz}, 1 \mathrm{H}), 6.80(\mathrm{t}, J=7.5 \mathrm{~Hz}, 1 \mathrm{H}), 6.88(\mathrm{t}, J=7.5 \mathrm{~Hz}, 1 \mathrm{H}), 7.19(\mathrm{~d}, J=6.0 \mathrm{~Hz}$, 
1H), $7.64(\mathrm{~d}, J=8.7 \mathrm{~Hz}, 2 \mathrm{H}), 7.79$ (d, $J=6.9 \mathrm{~Hz}, 1 \mathrm{H}), 7.82(\mathrm{~d}, J=6.9 \mathrm{~Hz}, 1 \mathrm{H}), 7.95$ (d, $J=8.4 \mathrm{~Hz}$, 1H). ${ }^{13} \mathrm{C}$ NMR (75 MHz, CDCl3) $\delta_{\mathrm{C}}: 13.5,29.4,30.6,46.1,52.7,60.5,64.2,66.3,81.7$, $115.7,117.6,119.4,123.0,124.6,124.8,125.7,125.8,127.2,129.6,130.6,132.1$, $134.2,134.4,140.9,162.4,169.0,205.5$

Methyl [spiro[3',2"]-acenapthene-1"-one]-3-oxo-3,3',4,5',7',7a'-hexahydro-1'H-spiro[benzo [b][1,4] thiazine-2,6'-pyrrolo[1,2-c]thiazole]-7'-carboxylate (12a): Obtained as pale white solid; Yield 74\%; m.p. 220-222 ${ }^{\circ} \mathrm{C}$; Anal. Calcd. for $\mathrm{C}_{26} \mathrm{H}_{20} \mathrm{~N}_{2} \mathrm{O}_{3} \mathrm{~S}: \mathrm{C}, 63.92 ; \mathrm{H}, 4.13$; N, 5.73; S, 13.13. Found: C, 63.84; H, 4.18; N, 5.78; S, 13.06. ${ }^{1} \mathrm{H}$ NMR (300 MHz, DMSO$\left.\mathrm{D}_{6} / \mathrm{CDCl}_{3}\right) \delta_{\mathrm{H}}: 3.12(\mathrm{~d}, \mathrm{~J}=7.5 \mathrm{~Hz}, 1 \mathrm{H}), 3.32-3.38(\mathrm{~m}, 1 \mathrm{H}), 3.42(\mathrm{~d}, J=8.7 \mathrm{~Hz}, 1 \mathrm{H}), 3.76-3.83$ (m, 4H), 4.62 (d, J=10.2Hz, 1H), 4.89 (d, J=4.2 Hz, 1H), 5.89 (d, J=7.8 Hz, 1H), 6.70 (t, $J=7.5 \mathrm{~Hz}, 1 \mathrm{H}), 6.81(\mathrm{t}, J=7.2 \mathrm{~Hz}, 1 \mathrm{H}), 7.11(\mathrm{~d}, J=7.5 \mathrm{~Hz}, 1 \mathrm{H}), 7.27$ (d, $J=6.6 \mathrm{~Hz},, 1 \mathrm{H}), 7.43-$ $7.48(\mathrm{~m}, 1 \mathrm{H}), 7.57-7.62(\mathrm{~m}, 1 \mathrm{H}), 7.82(\mathrm{~d}, J=8.4 \mathrm{~Hz}, 1 \mathrm{H}), 7.95$ (t, $J=8.7 \mathrm{~Hz}, 2 \mathrm{H}), 9.51(\mathrm{~s}, 1 \mathrm{H})$. ${ }^{13} \mathrm{C}$ NMR (75 MHz, CDCl3) $\delta_{\mathrm{C}}: 34.6,50.0,51.2,62.7,66.6,79.1,114.9,116.0,119.1,122.0$, $123.2,124.8,126.1,126.5,126.7,127.2,128.5,129.7,130.2,132.2,135.5,139.3,161.3$, 168.1, 199.6.

Ethyl [spiro[3',2"]-acenapthene-1"'-one]-3-oxo-3,3',4,5',7',7a'-hexahydro-1'H-spiro[benzo[b] [1,4]thiazine-2,6'-pyrrolo[1,2-c]thiazole]-7'-carboxylate (12b): Obtained as pale white solid; Yield 71\%; m.p. $222-224^{\circ} \mathrm{C}$; Anal. Calcd. for $\mathrm{C}_{27} \mathrm{H}_{22} \mathrm{~N}_{2} \mathrm{O}_{4} \mathrm{~S}_{2}$ : C, 64.52; H, 4.41; N, 5.57; S, 12.76. Found: C, 64,59; H, 4.34; N, 5.63; S, 12.68. ${ }^{1} \mathrm{H}$ NMR (300 MHz, DMSO-D $\left.6 / \mathrm{CDCl}_{3}\right)$ $\delta_{\mathrm{H}}: 1.28(\mathrm{t}, J=7.2 \mathrm{~Hz}, 3 \mathrm{H}), 3.30-3.36(\mathrm{~m}, 1 \mathrm{H}), 3.42(\mathrm{~d}, J=8.7 \mathrm{~Hz}, 1 \mathrm{H}), 3.77(\mathrm{~d}, J=8.7 \mathrm{~Hz}, 1 \mathrm{H})$, $4.23(\mathrm{dd}, J=14.1,7.2 \mathrm{~Hz}, 2 \mathrm{H}), 4.59(\mathrm{~d}, J=10.2 \mathrm{~Hz}, 1 \mathrm{H}), 4.83-4.9(\mathrm{~m}, 1 \mathrm{H}), 5.84(\mathrm{~d}, J=7.8 \mathrm{~Hz}$, $1 \mathrm{H}), 6.68(\mathrm{t}, J=7.8 \mathrm{~Hz}, 1 \mathrm{H}), 6.79(\mathrm{t}, J=7.5 \mathrm{~Hz}, 1 \mathrm{H}), 7.08(\mathrm{~d}, J=7.5 \mathrm{~Hz}, 1 \mathrm{H}), 7.26(\mathrm{~d}, J=6.9 \mathrm{~Hz}$ 1H), $7.46(\mathrm{t}, J=7.5 \mathrm{~Hz}, 1 \mathrm{H}), 7.59-7.64(\mathrm{~m}, 1 \mathrm{H}), 7.83(\mathrm{~d}, \mathrm{~J}=8.4 \mathrm{~Hz}, 1 \mathrm{H}), 7.91(\mathrm{~d}, J=7.2 \mathrm{~Hz}, 1 \mathrm{H})$, $7.98(\mathrm{~d}, J=8.1 \mathrm{~Hz}, 1 \mathrm{H}), 9.74(\mathrm{~s}, 1 \mathrm{H}) .{ }^{13} \mathrm{C} \mathrm{NMR}(75 \mathrm{MHz}, \mathrm{CDCl} 3) \delta_{\mathrm{C}}: 13.3,35.0,38.1,50.5$, 
$51.6,60.5,63.0,66.9,79.6,115.2,116.5,119.4,122.3,123.5,125.0,126.4,126.8,126.9$, $127.6,128.8,130.0,130.4,132.4,135.6,139.6,161.7,167.8,200.1$.

Methyl 1'-methyl-[spiro[2',3"]-oxindole]-3-oxo-3,4-dihydrospiro[benzo[b][1,4]thiazine-2,3'pyrrolidine]-4'-carboxylate (14a): Obtained as colourless solid; Yield 71\%; m.p. 266-268 ${ }^{\circ}$; Anal. Calcd. for $\mathrm{C}_{21} \mathrm{H}_{19} \mathrm{~N}_{3} \mathrm{O}_{4} \mathrm{~S}$ : C, 61.60; H, 4.68; N, 10.26; S, 7.83. Found: C, 61.67; H, 4.61; N, 10.29; S, 7.75. ${ }^{1} \mathrm{H}$ NMR (300 MHz, DMSO-D $\left./ \mathrm{CDCl}_{3}\right) \delta_{\mathrm{H}}: 3.11(\mathrm{~s}, 3 \mathrm{H}), 3.31(\mathrm{t}$, $J=11.2 \mathrm{~Hz}, 1 \mathrm{H}), 3.74(\mathrm{~s}, 3 \mathrm{H}), 4.07(\mathrm{t}, J=9.3 \mathrm{~Hz}, 1 \mathrm{H}), 4.82-4.89(\mathrm{~m}, 1 \mathrm{H}), 6.33(\mathrm{~d}, J=7.5 \mathrm{~Hz}$, $1 \mathrm{H}), 6.29(\mathrm{~d}, J=6.9 \mathrm{~Hz}, 1 \mathrm{H}), 6.71-6.78(\mathrm{~m}, 2 \mathrm{H}), 6.80-6.91(\mathrm{~m}, 2 \mathrm{H}), 7.05(\mathrm{~d}, J=6.9 \mathrm{~Hz}, 1 \mathrm{H})$, 7.13-7.15 (m, 1H), $9.56(\mathrm{~s}, 1 \mathrm{H}), 10.30(\mathrm{~s}, 1 \mathrm{H}) . \mathrm{C}^{13} \mathrm{NMR}\left(75 \mathrm{MHz}, \mathrm{CDCl}_{3}\right) \delta_{\mathrm{C}}: 33.8,44.8$, $50.4,52.1,55.4,76.6,107.4,114.1,115.2,119.8,121.4,124.4,125.8,128.1,135.1,141.8$, $164.5,169.0,173.5$.

Ethyl 1'-methyl-[spiro[2',3"]-oxindole]-3-oxo-3,4-dihydrospiro[benzo[b][1,4]thiazine-2,3'pyrrolidine]-4'-carboxylate (14b): Obtained as pale white solid; Yield 70\%; m.p. 279-281 ${ }^{\circ} \mathrm{C}$; Anal. Calcd. for $\mathrm{C}_{22} \mathrm{H}_{21} \mathrm{~N}_{3} \mathrm{O}_{4} \mathrm{~S}: \mathrm{C}, 62.40 ; \mathrm{H}, 5.00 ; \mathrm{N}, 9.92$, S, 7.57. Found: C, 62.32; H, 4.93; N, 9.98; S, 7.49. ${ }^{1} \mathrm{H}$ NMR (300 MHz, DMSO-D $\left./ \mathrm{CDCl}_{3}\right) \delta_{\mathrm{H}}: 1.29(\mathrm{t}, J=7.2 \mathrm{~Hz}, 3 \mathrm{H}), 2.05$ (s, $3 \mathrm{H}), 3.32(\mathrm{t}, J=8.7 \mathrm{~Hz}, 1 \mathrm{H}), 4.06(\mathrm{t}, J=9.0 \mathrm{~Hz}, 1 \mathrm{H}), 4.21(\mathrm{dd}, J=14.4,7.2 \mathrm{~Hz}, 2 \mathrm{H}), 4.81(\mathrm{t}$, $J=8.7 \mathrm{~Hz}, 1 \mathrm{H}), 6.22(\mathrm{~d}, J=7.5 \mathrm{~Hz}, 1 \mathrm{H}), 6.28(\mathrm{~d}, J=7.8 \mathrm{~Hz}, 1 \mathrm{H}), 6.75(\mathrm{t}, J=6.3 \mathrm{~Hz}, 2 \mathrm{H}), 6.79-$ $6.88(\mathrm{~m}, 2 \mathrm{H}), 7.06(\mathrm{~d}, J=7.2 \mathrm{~Hz}, 1 \mathrm{H}), 7.14(\mathrm{~d}, J=6.9 \mathrm{~Hz}, 1 \mathrm{H}), 9.52(\mathrm{~s}, 1 \mathrm{H}), 10.26(\mathrm{~s}, 1 \mathrm{H}) .{ }^{13} \mathrm{C}$ $\operatorname{NMR}\left(75 \mathrm{MHz}, \mathrm{CDCl}_{3}\right) \delta_{\mathrm{C}}: 12.8,33.7,44.6,52.0,55.2,59.2,76.6,107.2,113.9,115.2$, $119.6,121.1,123.2,124.3,125.6,128.0,135.0,141.7,164.4,168.3,173.3$.

Methyl 1'-methyl-[spiro[2',3"]-5-chlorooxindole]-3-oxo-3,4-dihydrospiro[benzo[b][1,4] thiazine-2,3'-pyrrolidine]-4'-carboxylate (14c): Obtained as colourless solid; Yield 81\%; m.p. 272-274 ${ }^{\circ} \mathrm{C}$; Anal. Calcd. for $\mathrm{C}_{21} \mathrm{H}_{18} \mathrm{ClN}_{3} \mathrm{O}_{4} \mathrm{~S}$ : C, 56.82; H, 4.09; N, 9.47; S, 7.22. Found: C, 56.76; H, 4.03; N, 9.40; S, 7.18. ${ }^{1} \mathrm{H}$ NMR (300 MHz, DMSO-D $\left.6 / \mathrm{CDCl}_{3}\right) \delta_{\mathrm{H}}: 2.23(\mathrm{~s}, 3 \mathrm{H})$, $3.50(\mathrm{t}, J=8.7 \mathrm{~Hz}, 1 \mathrm{H}), 3.70(\mathrm{~s}, 1 \mathrm{H}), 3.91-3.95(\mathrm{~m}, 3 \mathrm{H}), 4.20(\mathrm{t}, J=9.3 \mathrm{~Hz}, 1 \mathrm{H}), 4.95-5.01(\mathrm{~m}$, 
1H), 6.34-6.38 (m, 1H), 6.55-6.57 (m, 1H), 6.95-6.98 (m, 1H), $7.11(\mathrm{~d}, J=8.1 \mathrm{~Hz}, 2 \mathrm{H}), 7.22$ $(\mathrm{d}, J=2.1 \mathrm{~Hz}, 1 \mathrm{H}), 7.30-7.32(\mathrm{~m}, 1 \mathrm{H}), 9.93(\mathrm{~s}, 1 \mathrm{H}), 10.81(\mathrm{~s}, 1 \mathrm{H}) .{ }^{13} \mathrm{C} \mathrm{NMR}\left(75 \mathrm{MHz}, \mathrm{CDCl}_{3}\right)$ $\delta_{\mathrm{C}}: 34.4,45.2,50.9,52.7,56.0,76.6,109.0,114.7,115.7,121.9,123.9,125.4,126.4,126.6$, $126.8,128.4,135.6,140.9,165.0,169.4,173.8$.

Ethyl 1'-methyl-[spiro[2',3"]-5-chlorooxindole]-3-oxo-3,4-dihydrospiro[benzo[b][1,4] thiazine-2,3'-pyrrolidine]-4'-carboxylate (14d): Obtained as colourless solid; Yield 82\%; m.p. $289-291^{\circ} \mathrm{C}$; Anal. Calcd. for $\mathrm{C}_{22} \mathrm{H}_{20} \mathrm{ClN}_{3} \mathrm{O}_{4} \mathrm{~S}: \mathrm{C}, 57.70 ; \mathrm{H}, 4.40 ; \mathrm{Cl}, 7.74 ; \mathrm{N}, 9.18 ; \mathrm{S}$, 7.00. Found: C, 57.77; H, 4.32; Cl, 7.81; N, 9.23; S, 7.06. ${ }^{1} \mathrm{H}$ NMR (300 MHz, DMSO$\left.\mathrm{D}_{6} / \mathrm{CDCl}_{3}\right) \delta_{\mathrm{H}}: 2.08(\mathrm{~s}, 3 \mathrm{H}), 3.34-3.40(\mathrm{~m}, 1 \mathrm{H}), 4.04(\mathrm{t}, J=9.0 \mathrm{~Hz}, 1 \mathrm{H}), 4.22(\mathrm{dd}, J=13.1$, $7.2 \mathrm{~Hz}, 2 \mathrm{H}), 4.78(\mathrm{t}, J=9.0 \mathrm{~Hz}, 1 \mathrm{H}), 6.19(\mathrm{~d}, J=7.1 \mathrm{~Hz}, 1 \mathrm{H}), 6.38-6.41(\mathrm{~m}, 1 \mathrm{H}), 6.76-6.79(\mathrm{~m}$, 2H), 6.84-6.87 (m, 1H), 7.13-7.16 (m, 1H), $9.47(\mathrm{~s}, 1 \mathrm{H}), 10.40(\mathrm{~s}, 1 \mathrm{H}) .{ }^{13} \mathrm{C}$ NMR $(75 \mathrm{MHz}$, $\left.\mathrm{CDCl}_{3}\right) \delta_{\mathrm{C}}: 13.7,29.0,34.7,45.6,53.2,56.3,60.4,78.4,109.2,115.0,116.3,122.2,124.3$, $125.9,126.7,126.9,127.2,128.8,136.0,141.2,165.5,169.3,171.3$.

Methyl [spiro[2',3"]-oxindole]-3-oxo-5'-phenyl-3,4-dihydrospiro[benzo[b][1,4]thiazine-2,3'pyrrolidine]-4'-carboxylate (15a): Obtained as pale white solid; Yield 99\%; m.p. 228-230 ${ }^{\circ}$; Anal. Calcd. for $\mathrm{C}_{26} \mathrm{H}_{21} \mathrm{~N}_{3} \mathrm{O}_{4} \mathrm{~S}: \mathrm{C}, 66.23 ; \mathrm{H}, 4.49 ; \mathrm{N}, 8.91 ; \mathrm{S}, 6.80$. Found: C, 66.31; H, 4.41; N, 8.97; S, 6.73. ${ }^{1} \mathrm{H}$ NMR (300 MHz, DMSO-D $\left./ \mathrm{CDCl}_{3}\right) \delta_{\mathrm{H}}: 2.81(\mathrm{~d}, J=6.9 \mathrm{~Hz}, 1 \mathrm{H}), 3.67$ (s, $3 \mathrm{H}), 4.75(\mathrm{~d}, J=9.9 \mathrm{~Hz}, 1 \mathrm{H}), 5.58-5.64(\mathrm{~m}, 1 \mathrm{H}), 6.31(\mathrm{~d}, J=7.5 \mathrm{~Hz}, 1 \mathrm{H}), 6.34-6.37(\mathrm{~m}, 1 \mathrm{H})$, 6.84-6.88 (m, 3H), 7.17-7.24 (m, 3H), 7.28-7.34 (m, 3H), 7.61 (d, J=7.5Hz, 2H), $9.64(\mathrm{~s}$, 1H), 10.35 (s, 1H). ${ }^{13} \mathrm{C}$ NMR:50.1, 55.3, 56.0, 59.7, 73.0, 107.0, 113.9, 114.9, 115.7, 119.3, $121.1,121.8,123.0,125.4,125.6,125.8,126.1,126.4,126.7,127.7,134.9,139.5,140.8$, $164.2,168.2,175.9$.

Ethyl [spiro[2',3"]-oxindole]-3-oxo-5'-phenyl-3,4-dihydrospiro[benzo[b][1,4]thiazine-2,3'pyrrolidine]-4'-carboxylate (15b): Obtained as colourless solid; Yield 73\%; m.p. 218-220 ${ }^{\circ}$; Anal. Calcd. for $\mathrm{C}_{27} \mathrm{H}_{23} \mathrm{~N}_{3} \mathrm{O}_{4} \mathrm{~S}$ : C, 66.79; H, 4.77; N, 8.65; S, 6.60. Found: C, C, 66.71; H, 
4.82; N, 8.57; S, 6.67. ${ }^{1} \mathrm{H}$ NMR (300 MHz, DMSO-D $\left.6 / \mathrm{CDCl}_{3}\right) \delta_{\mathrm{H}}: 1.21(\mathrm{t}, J=7.2 \mathrm{~Hz}, 3 \mathrm{H})$, $2.73(\mathrm{~d}, J=7.5 \mathrm{~Hz}, 1 \mathrm{H}), 4.10-4.18(\mathrm{~m}, 2 \mathrm{H}), 4.71(\mathrm{~d}, J=9.9 \mathrm{~Hz}, 1 \mathrm{H}), 6.00(\mathrm{~m}, 1 \mathrm{H}), 6.31(\mathrm{~d}$, $J=7.5 \mathrm{~Hz}, 1 \mathrm{H}), 6.35-6.38(\mathrm{~m}, 1 \mathrm{H}), 6.82-6.88(\mathrm{~m}, 3 \mathrm{H}), 6.95(\mathrm{t}, J=7.8 \mathrm{~Hz}, 1 \mathrm{H}), 7.18-7.24(\mathrm{~m}$, 1H), 7.29-7.37 (m, 3H), $7.62(\mathrm{~d}, J=7.2 \mathrm{~Hz}, 2 \mathrm{H}), 7.67$ (s, 1H), $9.58(\mathrm{~s}, 1 \mathrm{H}), 10.27(\mathrm{~s}, 1 \mathrm{H}) .{ }^{13} \mathrm{C}$ NMR (75 MHz, CDCl3) $\delta_{\mathrm{C}}: 13.1,56.0,57.5,60.0,61.1,73.7,108.0,114.7,120.3,121.9$, $123.7,126.3,126.5,126.7,126.8,127.0,127.4,128.5,135.5,139.8,141.0,164.8,168.4$, 176.6.

Methyl [spiro[2',3"]-oxindole]-3-oxo-5'-phenyl-3,4-dihydrospiro[benzo[b][1,4]thiazine-2,3'pyrrolidine]-4'-carboxylate (15c): Obtained as pale white solid; Yield 99\%; m.p. 212$214^{\circ} \mathrm{C}$; Anal. Calcd. for $\mathrm{C}_{26} \mathrm{H}_{20} \mathrm{ClN}_{3} \mathrm{O}_{4} \mathrm{~S}: \mathrm{C}, 61.72 ; \mathrm{H}, 3.98 ; \mathrm{Cl}, 7.01 ; \mathrm{N}, 8.30 ; \mathrm{S}, 6.34$. Found: C, 61.80; H, 3.91; Cl, 7.08; N, 8.37; S, 6.34. ${ }^{1} \mathrm{H}$ NMR (300 MHz, DMSO-D $\left.6 / \mathrm{CDCl}_{3}\right)$ $\delta_{\mathrm{H}}: 2.81(\mathrm{~d}, J=6.9 \mathrm{~Hz}, 1 \mathrm{H}), 3.66(\mathrm{~s}, 3 \mathrm{H}), 3.80(\mathrm{~s}, 1 \mathrm{H}), 4.75(\mathrm{~d}, J=9.9 \mathrm{~Hz}, 1 \mathrm{H}), 5.58-5.63(\mathrm{~m}$, $1 \mathrm{H}), 6.29(\mathrm{~d}, J=7.5 \mathrm{~Hz}, 1 \mathrm{H}), 6.35-6.37(\mathrm{~m}, 1 \mathrm{H}), 6.83-6.88(\mathrm{~m}, 3 \mathrm{H}), 6.93(\mathrm{~d}, J=7.3 \mathrm{~Hz}, 1 \mathrm{H})$, 7.17-7.23 (m, 3H), 7.28-7.34 (m, 3H), $7.61(\mathrm{~d}, J=7.5 \mathrm{~Hz}, 2 \mathrm{H}), 9.63(\mathrm{~s}, 1 \mathrm{H}), 10.35(\mathrm{~s}, 1 \mathrm{H}) .{ }^{13} \mathrm{C}$ NMR (75 MHz, CDCl3) $\delta_{\mathrm{C}}: 50.1,55.3,56.0,59.7,73.0,107.0,113.9,114.9,115.7,119.3$, $121.1,121.8,123.0,125.4,125.6,125.8,126.1,126.4,126.7,127.7,134.9,139.5,139.5$, $140.8,164.2,168.2,175.8$.

Ethyl [spiro[2',3"]-5-chlorooxindole]-3-oxo-5'-phenyl-3,4-dihydrospiro[benzo[b][1,4] thiazine-2,3'-pyrrolidine]-4'-carboxylate (15d): Obtained as pale white solid; Yield 98\%; m.p. $240-242^{\circ} \mathrm{C}$; Anal. Calcd. for $\mathrm{C}_{27} \mathrm{H}_{22} \mathrm{ClN}_{3} \mathrm{O}_{4} \mathrm{~S}$ : C, 62.36; H, 4.26; N, 8.08; S, 6.17. Found: C, 62.44; H, 4.19; N, 8.02; S, 6.24. ${ }^{1} \mathrm{H}$ NMR (300 MHz, DMSO-D $\left.6 / \mathrm{CDCl}_{3}\right) \delta_{\mathrm{H}}: 1.20$ $(\mathrm{t}, J=7.2 \mathrm{~Hz}, 3 \mathrm{H}), 4.08-4.14(\mathrm{~m}, 2 \mathrm{H}), 4.60(\mathrm{~d}, J=9.3 \mathrm{~Hz}, 1 \mathrm{H}), 5.55(\mathrm{dd}, J=9.6,6.3 \mathrm{~Hz}, 1 \mathrm{H})$, $6.21(\mathrm{~d}, . J=5.1 \mathrm{~Hz}, 1 \mathrm{H}), 6.39-6.41(\mathrm{~m}, 1 \mathrm{H}), 6.84-6.92(\mathrm{~m}, 3 \mathrm{H}), 7.15-7.20(\mathrm{~m}, 2 \mathrm{H}), 7.27-7.37$ (m, 3H), $7.58(\mathrm{~d}, J=7.5 \mathrm{~Hz}, 2 \mathrm{H}), 9.87(\mathrm{~s}, 1 \mathrm{H}), 10.7$ (s, 1H). ${ }^{13} \mathrm{C} \mathrm{NMR}(75 \mathrm{MHz}, \mathrm{CDCl} 3) \delta_{\mathrm{C}}$ : 
$12.6,27.8,55.1,56.0,59.1,59.8,72.9,108.2,114.1,115.1,121.2,123.4,124.1,125.9,126.0$, $126.2,126.7,127.4,128.6,135.1,139.5,139.7,164.1,167.6,175.9$.

Methyl [spiro[5',3']-oxindole]-3-oxo-3,3',4,5',7',7a'-hexahydro-1'H-spiro[benzo[b][1,4] thiazine-2,6'-pyrrolo[1,2-c]thiazole]-7'-carboxylate (16a): Obtained as pale white solid; Yield 73\%; m.p. $252-254^{\circ} \mathrm{C}$; Anal. Calcd. for $\mathrm{C}_{22} \mathrm{H}_{19} \mathrm{~N}_{3} \mathrm{O}_{4} \mathrm{~S}_{2}$ : C, 58.26; H, 4.22; N, 9.27; S, 14.14. Found: C, 58.19; H, 4.27; N, 9.31; S, 14.07. ${ }^{1} \mathrm{H}$ NMR (300 MHz, DMSO-D $\left./ \mathrm{CDCl}_{3}\right)$ $\delta_{\mathrm{H}}: 2.59(\mathrm{t}, J=1.8 \mathrm{~Hz}, 1 \mathrm{H}), 2.96(\mathrm{dd}, J=10.5,5.4 \mathrm{~Hz}, 1 \mathrm{H}), 3.34(\mathrm{~d}, J=7.8 \mathrm{~Hz}, 1 \mathrm{H}), 3.64(\mathrm{~d}$, $J=7.8 \mathrm{~Hz}, 1 \mathrm{H}), 3.74(\mathrm{~s}, 3 \mathrm{H}), 4.54(\mathrm{~d}, J=10.2 \mathrm{~Hz}, 1 \mathrm{H}) .4 .77-4.85(\mathrm{~m}, 1 \mathrm{H}), 6.50$ (dd, $J=11.1,7.2 \mathrm{~Hz}, 2 \mathrm{H}), 6.86-6.92(\mathrm{~m}, 3 \mathrm{H}), 7.05(\mathrm{~d}, J=7.8 \mathrm{~Hz}, 1 \mathrm{H}), 7.18(\mathrm{~d}, J=7.5 \mathrm{~Hz}, 1 \mathrm{H}), 7.41$ (d, $J=7.5 \mathrm{~Hz}, 1 \mathrm{H}), 9.77(\mathrm{~s}, 1 \mathrm{H}), 10.37(\mathrm{~s}, 1 \mathrm{H}) .{ }^{13} \mathrm{C} \mathrm{NMR}(75 \mathrm{MHz}, \mathrm{CDCl} 3) \delta_{\mathrm{C}}: 32.5,47.5,49.7$, $50.6,61.0,65.3,73.4,107.7,114.4,115.2,119.4,121.3,122.6,125.3,125.7,126.2,128.3$, $135.0,141.1,161.6,167.7,173.1$.

Ethyl [spiro[5',3']-oxindole]-3-oxo-3,3',4,5',7',7a'-hexahydro-1'H-spiro[benzo[b][1,4] thiazine-2,6'-pyrrolo[1,2-c]thiazole]-7'-carboxylate (16b): Obtained as pale white solid; Yield 88\%; m.p. $271-273^{\circ} \mathrm{C}$; Anal. Calcd. for $\mathrm{C}_{23} \mathrm{H}_{21} \mathrm{~N}_{3} \mathrm{O}_{4} \mathrm{~S}_{2}$ : C, 59.08; H, 4.53; N, 8.99; S, 13.72. Found: C, 59.16; H, 4.46; N, 9.05; S, 13.65. ${ }^{1} \mathrm{H}$ NMR (300 MHz, DMSO-D $\left.6 / \mathrm{CDCl}_{3}\right)$ $\delta_{\mathrm{H}}: 1.21-1.30(\mathrm{~m}, 3 \mathrm{H}), 3.00(\mathrm{dd}, J=10.8,6.3 \mathrm{~Hz}, 2 \mathrm{H}), 3.24(\mathrm{dd}, J=10.8,6.3 \mathrm{~Hz}, 1 \mathrm{H}), 3.41(\mathrm{~d}$, $J=8.1 \mathrm{~Hz}, 1 \mathrm{H}), 3.72(\mathrm{~d}, J=8.1 \mathrm{~Hz}, 1 \mathrm{H}), 4.19-4.28(\mathrm{~m}, 2 \mathrm{H}), 4.52(\mathrm{~d}, J=10.2 \mathrm{~Hz}, 1 \mathrm{H}), 4.80-4.8$ $(\mathrm{m}, 1 \mathrm{H}), 6.54(\mathrm{~d}, J=7.8 \mathrm{~Hz}, 2 \mathrm{H}), 6.82-6.96(\mathrm{~m}, 3 \mathrm{H}), 7.06-7.11(\mathrm{~m}, 1 \mathrm{H}), 7.35(\mathrm{~d}, J=7.5 \mathrm{~Hz}, 1 \mathrm{H})$, $9.40(\mathrm{~s}, 1 \mathrm{H}), 10.0(\mathrm{~s}, 1 \mathrm{H}) .{ }^{13} \mathrm{C} \mathrm{NMR}(75 \mathrm{MHz}, \mathrm{CDCl} 3) \delta_{\mathrm{C}}: 13.6,34.7,50.1,51.4,60.8,62.2$, $66.6,75.1,108.9,115.5,116.8,120.4,122.4,123.2,126.6,127.2,127.4,129.4,135.8,141.7$, $161.9,168.2,173.9$.

Methyl [spiro[5',3"]-5-chlorooxindole]-3-oxo-3,3',4,5',7',7a'-hexahydro-1'H-spiro[benzo [b][1,4]thiazine-2,6'-pyrrolo[1,2-c]thiazole]-7'-carboxylate (16c): Obtained as pale white solid; Yield 76\%; m.p. 266-268 ${ }^{\circ}$; Anal. Calcd. for $\mathrm{C}_{22} \mathrm{H}_{18} \mathrm{ClN}_{3} \mathrm{O}_{4} \mathrm{~S}_{2}: \mathrm{C}, 54.15 ; \mathrm{H}, 3.72 ; \mathrm{N}$, 
8.61; S, 13.14. Found: C, $54.07 \mathrm{H}, 3.80 \mathrm{~N}, 8.68$; S, 13.05. ${ }^{1} \mathrm{H}$ NMR (300 MHz, DMSO$\left.\mathrm{D}_{6} / \mathrm{CDCl}_{3}\right) \delta_{\mathrm{H}}: 3.20(\mathrm{dd}, J=10.5,6.0 \mathrm{~Hz}, 1 \mathrm{H}), 3.37(\mathrm{~d}, J=8.1 \mathrm{~Hz}, 1 \mathrm{H}), 3.66(\mathrm{t}, J=7.8 \mathrm{~Hz}, 2 \mathrm{H})$, $3.76(\mathrm{~s}, 3 \mathrm{H}), 4.51(\mathrm{~d}, J=9.9 \mathrm{~Hz}, 1 \mathrm{H}), 4.77-4.82(\mathrm{~m}, 1 \mathrm{H}), 6.46(\mathrm{~d}, J=8.4 \mathrm{~Hz}, 1 \mathrm{H}), \quad 6.56(\mathrm{~d}$, $J=7.8 \mathrm{~Hz}, 1 \mathrm{H}), 6.84-6.89(\mathrm{~m}, 1 \mathrm{H}), 6.92-6.97(\mathrm{~m}, 1 \mathrm{H}), 7.06(\mathrm{dd}, J=8.1,1.8 \mathrm{~Hz}, 1 \mathrm{H}), 7.20(\mathrm{~d}$, $J=7.2 \mathrm{~Hz}, 1 \mathrm{H}), 7.45(\mathrm{~d}, J=1.8 \mathrm{~Hz}, 1 \mathrm{H}), 9.74(\mathrm{~s}, 1 \mathrm{H}), 10.42(\mathrm{~s}, 1 \mathrm{H}) .{ }^{13} \mathrm{C}$ NMR $(75 \mathrm{MHz}$, $\mathrm{CDCl3}) \delta_{\mathrm{C}}: 33.5,48.6,50.5,51.3,56.2,61.9,66.0,74.1,109.4,115.1,115.8,122.0,125.0$, $126.4,127.0,128.8,135.5,140.3,161.7,168.2,173.6$.

Ethyl [spiro[5',3']-5-chlorooxindole]-3-oxo-3,3',4,5',7',7a'-hexahydro-1'H-spiro[benzo[b] [1,4]thiazine-2,6'-pyrrolo[1,2-c]thiazole]-7'-carboxylate (16d): Obtained as pale white solid; Yield 94\%; m.p. 266-268 ${ }^{\circ}$; Anal. Calcd. for $\mathrm{C}_{23} \mathrm{H}_{20} \mathrm{ClN}_{3} \mathrm{O}_{4} \mathrm{~S}_{2}$ : C, 55.03; H, 4.02; N, 8.37; S, 12.77. Found: C, 55.09; H, 3.95; N, 8.29; S, 12.83. ${ }^{1} \mathrm{H}$ NMR (300 MHz, DMSO-D $\left.6 / \mathrm{CDCl}_{3}\right)$ $\delta_{\mathrm{H}}: 1.23-1.33(\mathrm{~m}, 3 \mathrm{H}), 2.95(\mathrm{dd}, J=7.2,6.0 \mathrm{~Hz}, 1 \mathrm{H}), 3.15-3.20(\mathrm{~m}, 2 \mathrm{H}), 3.36(\mathrm{~d}, J=10.5 \mathrm{~Hz}$, $1 \mathrm{H}), 3.63(\mathrm{~d}, J=7.8 \mathrm{~Hz}, 1 \mathrm{H}), 4.17-4.26(\mathrm{~m}, 2 \mathrm{H}), 4.58(\mathrm{~d}, J=9.9 \mathrm{~Hz}, 1 \mathrm{H}), 4.74-4.79(\mathrm{~m}, 1 \mathrm{H})$, $6.43(\mathrm{~d}, J=7.1 \mathrm{~Hz}, 1 \mathrm{H}), 6.54(\mathrm{~d}, J=7.8 \mathrm{~Hz}, 1 \mathrm{H}), 6.84-6.96(\mathrm{~m}, 2 \mathrm{H}), 7.03-7.06(\mathrm{~m}, 1 \mathrm{H}), 7.19$ (d, $J=7.5 \mathrm{~Hz}, 1 \mathrm{H}), 9.86(\mathrm{~s}, 1 \mathrm{H}), 10.52(\mathrm{~s}, 1 \mathrm{H}) .{ }^{13} \mathrm{C} \mathrm{NMR}(75 \mathrm{MHz}, \mathrm{CDCl} 3) \delta_{\mathrm{C}}: 13.2,28.5,33.6$, $48.7,50.5,60.4,61.9,66.0,74.2,109.4,115.1,115.9,122.0,124.9,125.0,126.4,127.0$, $128.8,135.6,140.3,161.8,167.6,173.7$. 


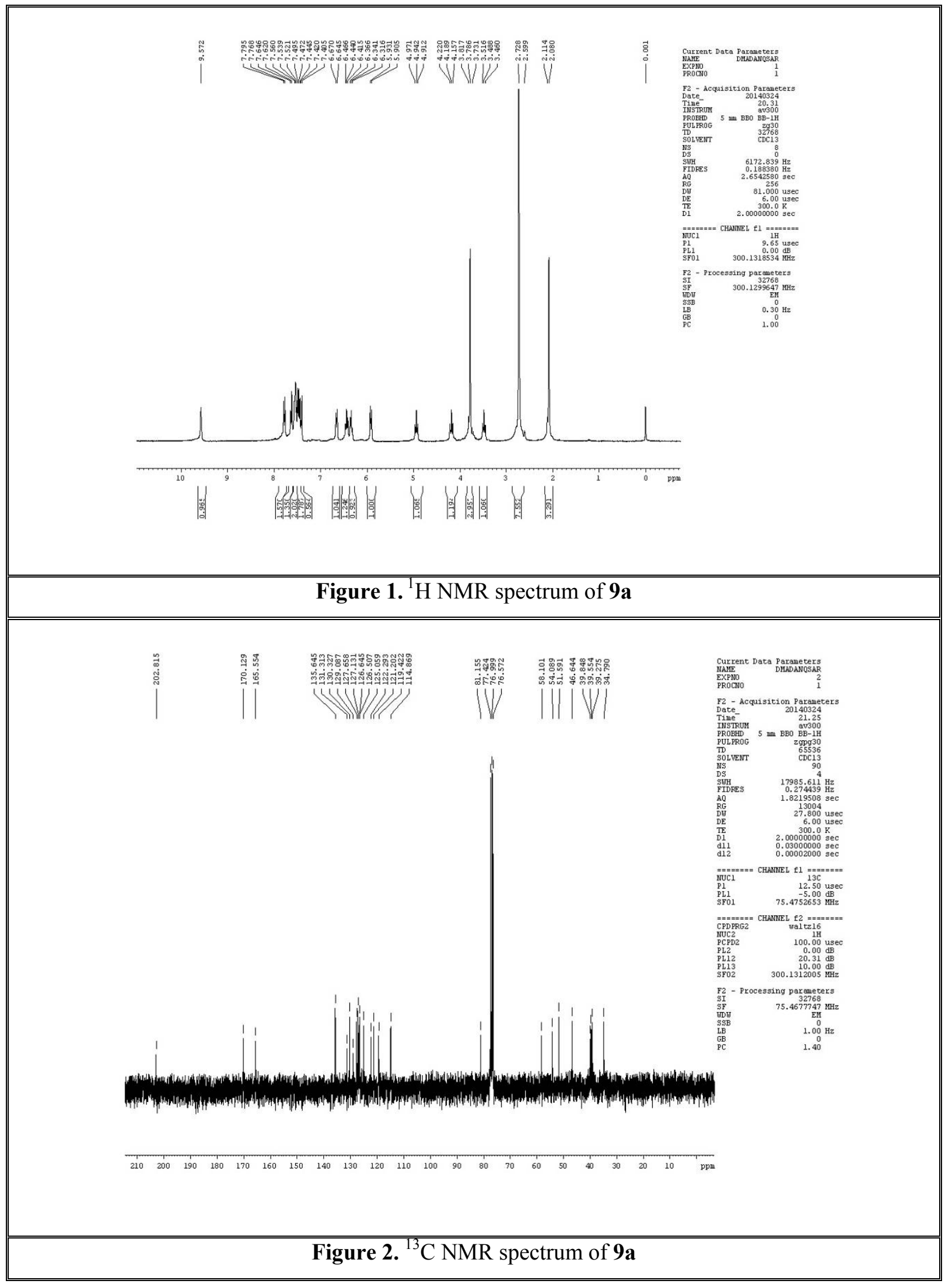




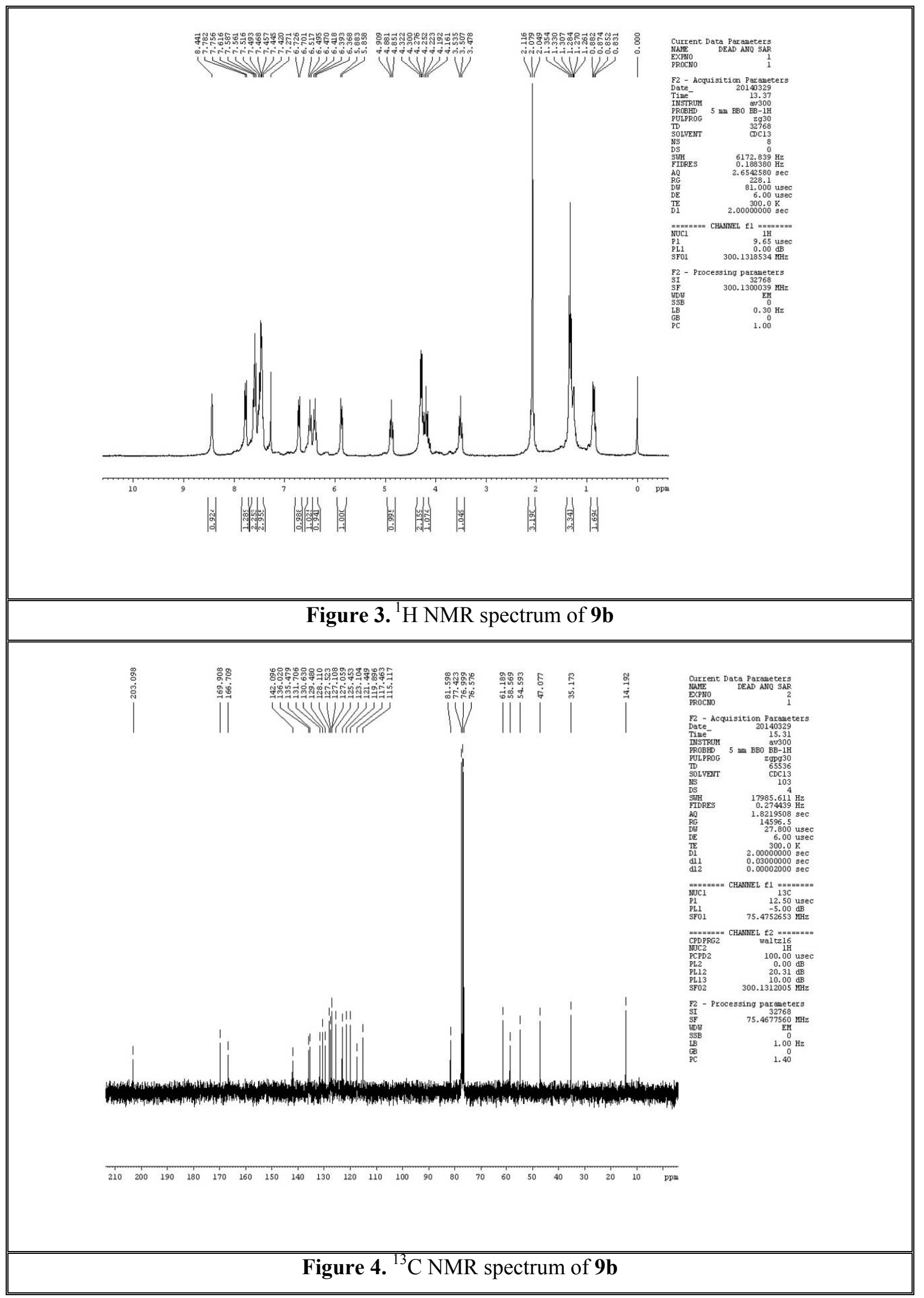




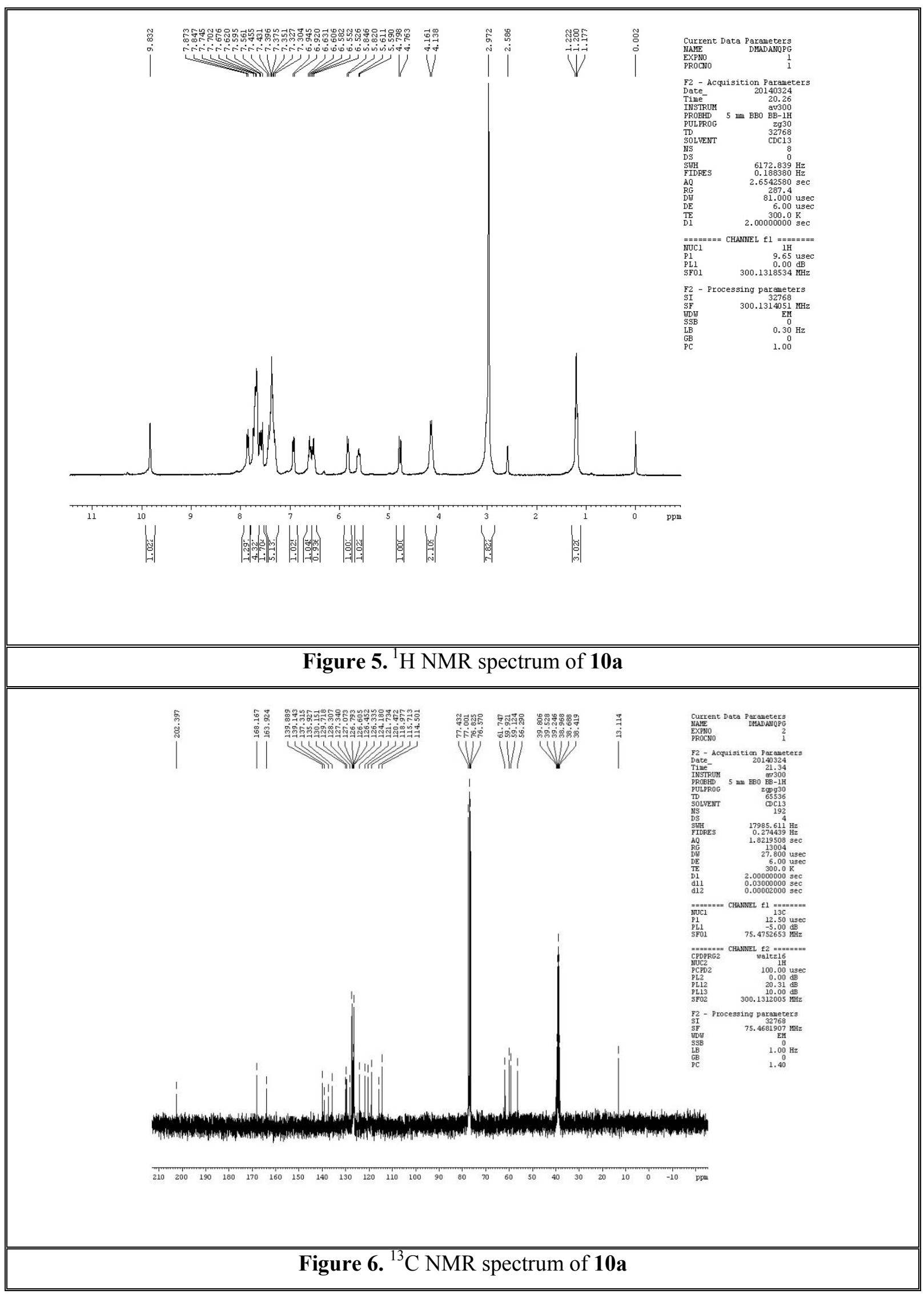




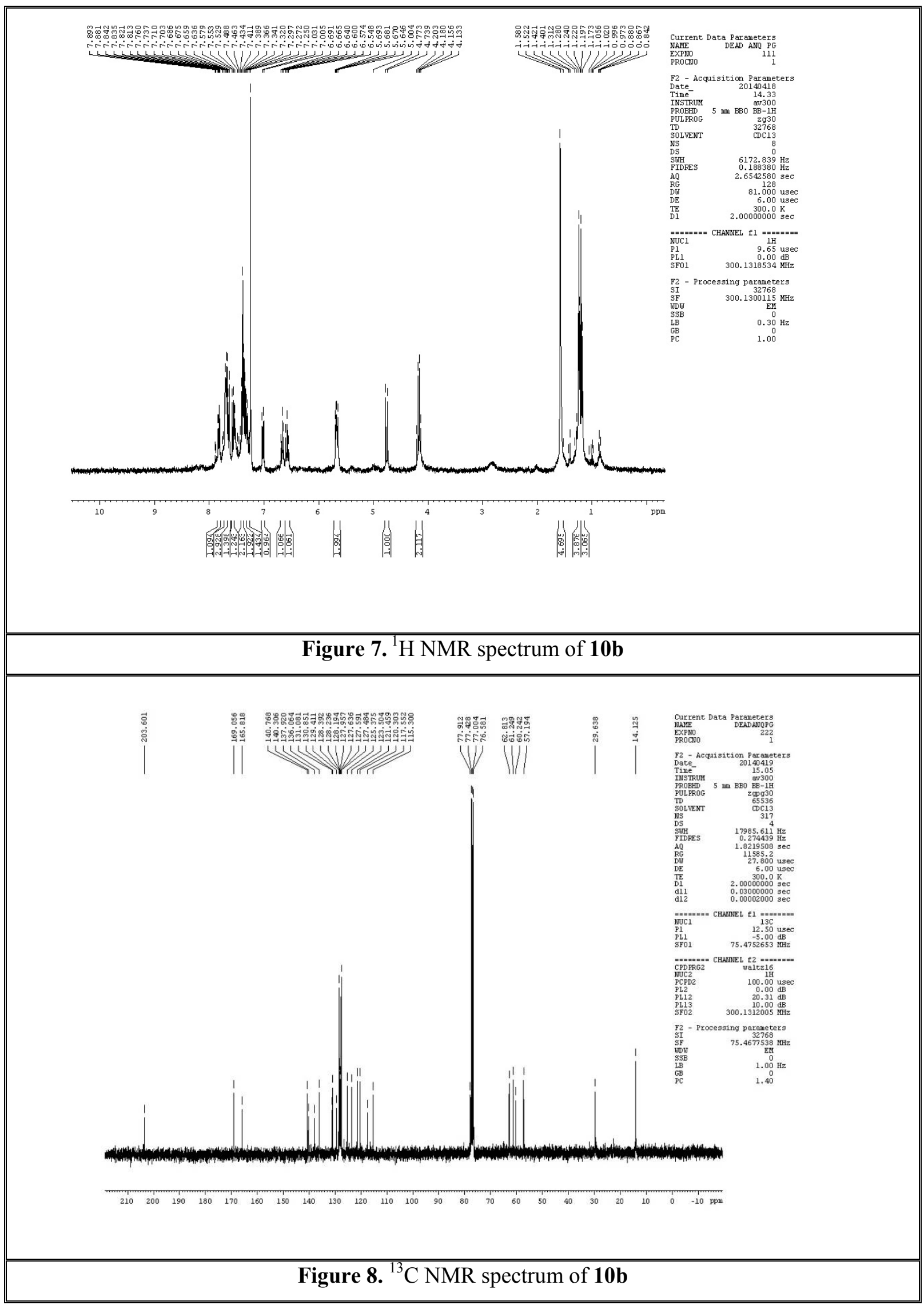




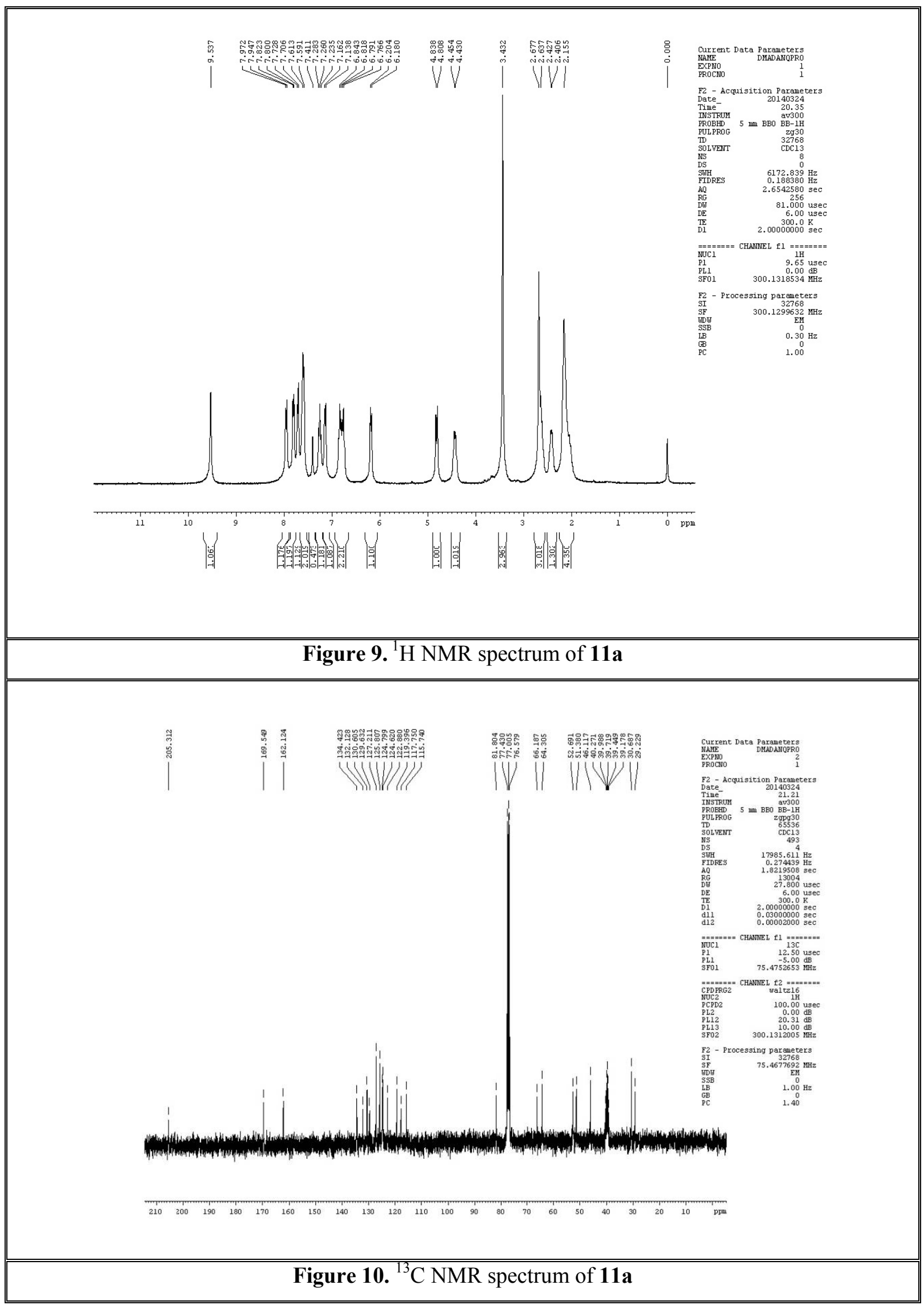




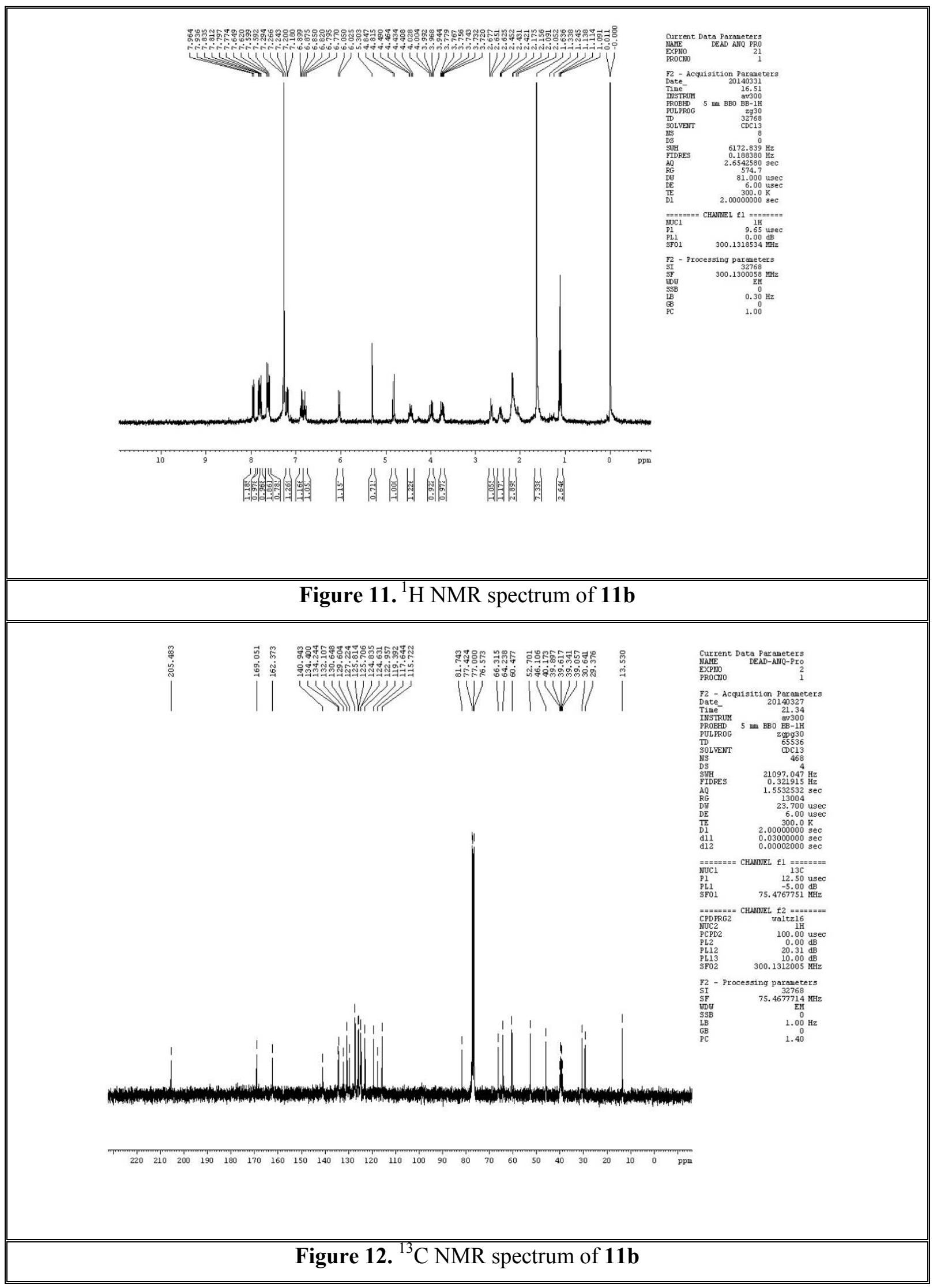




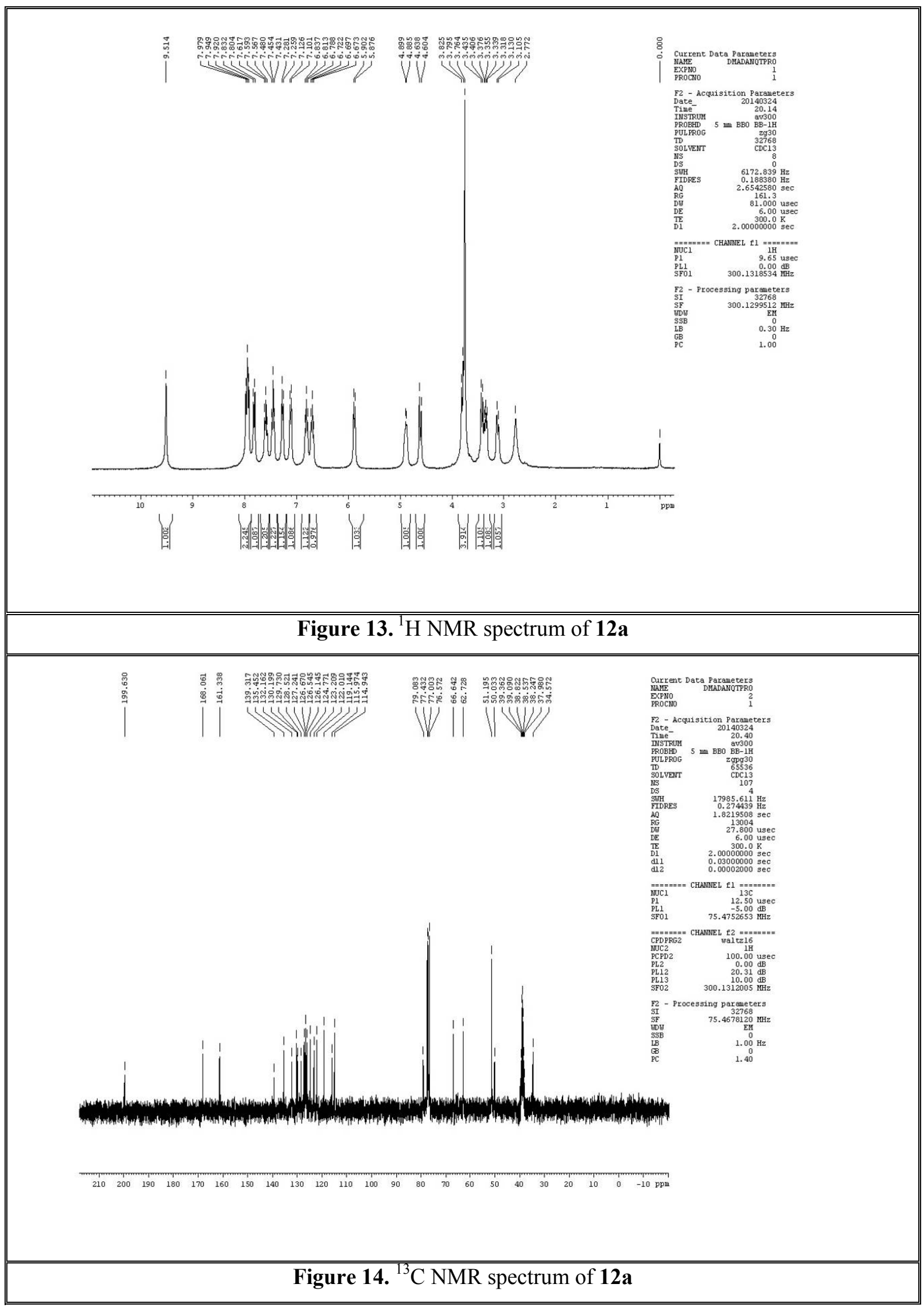




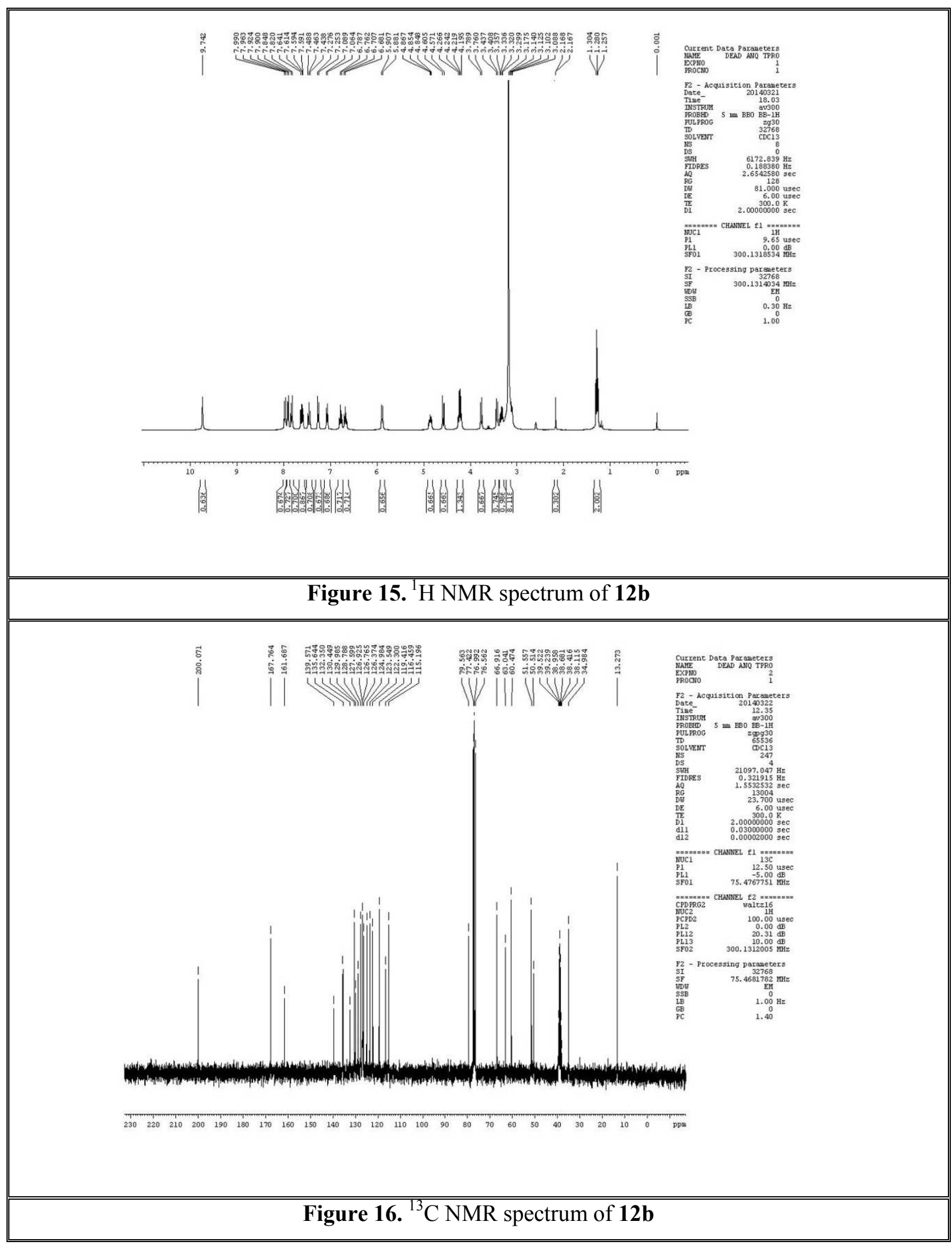




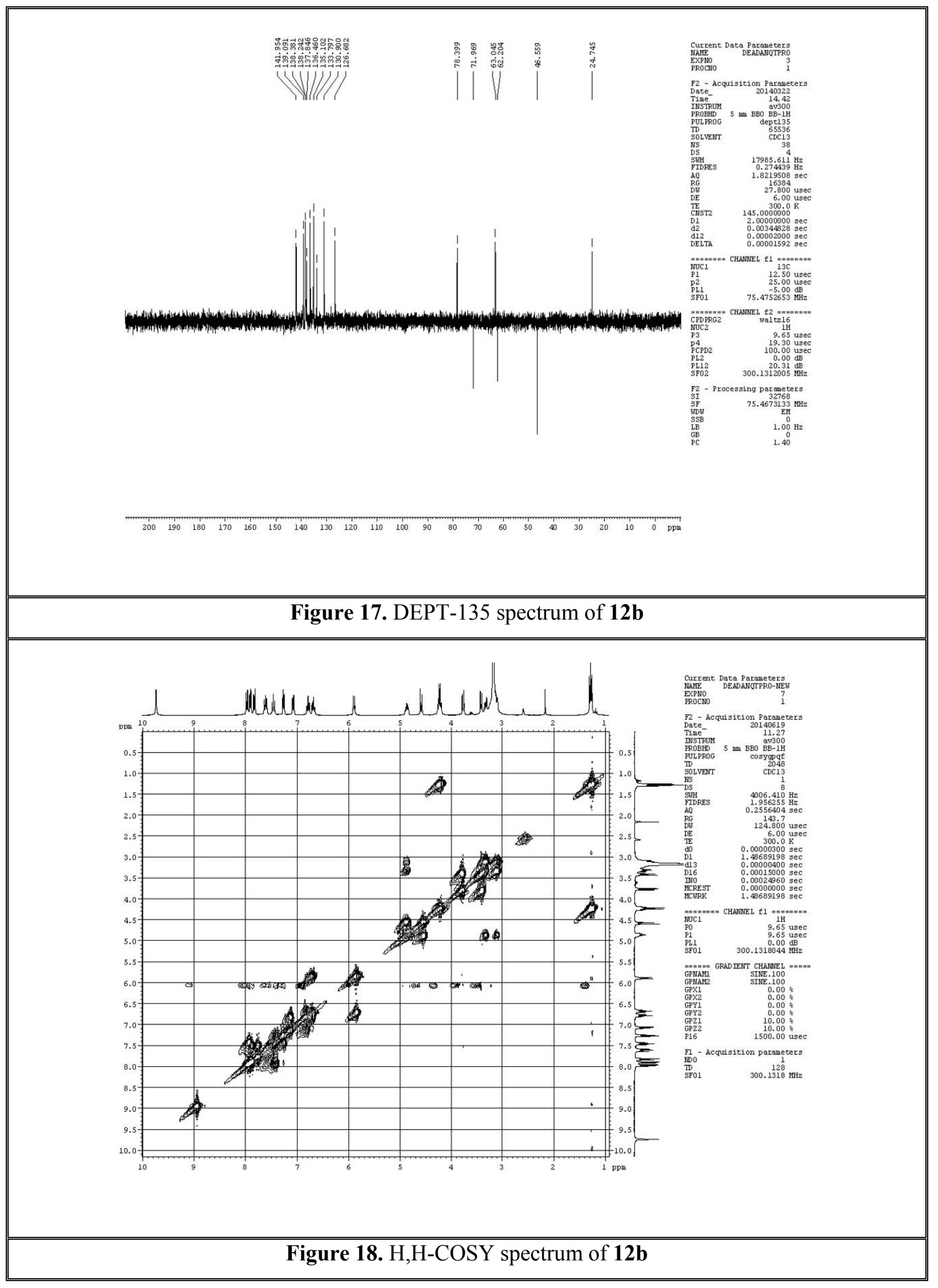




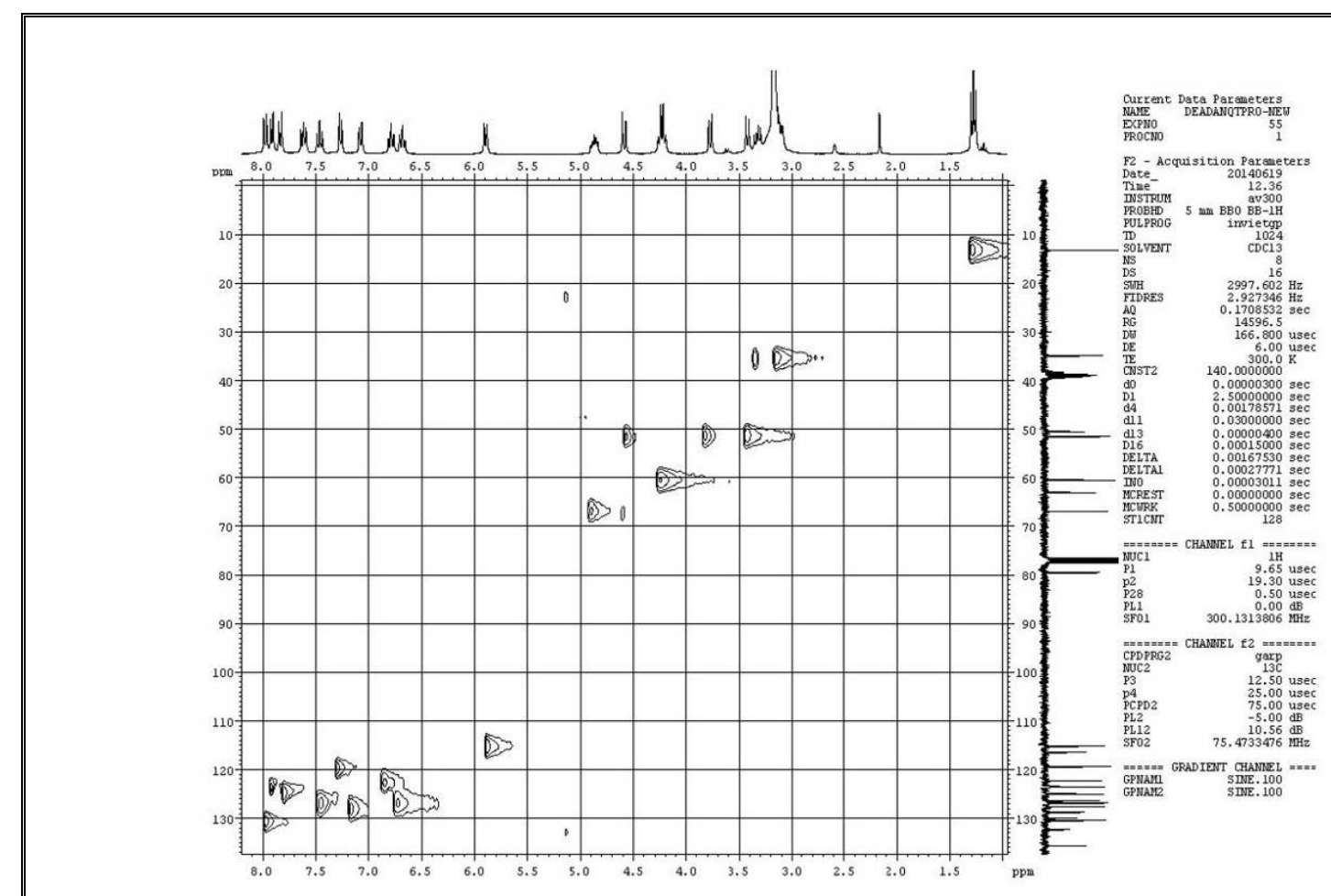

Figure 19. C,H-COSY spectrum of $\mathbf{1 2 b}$

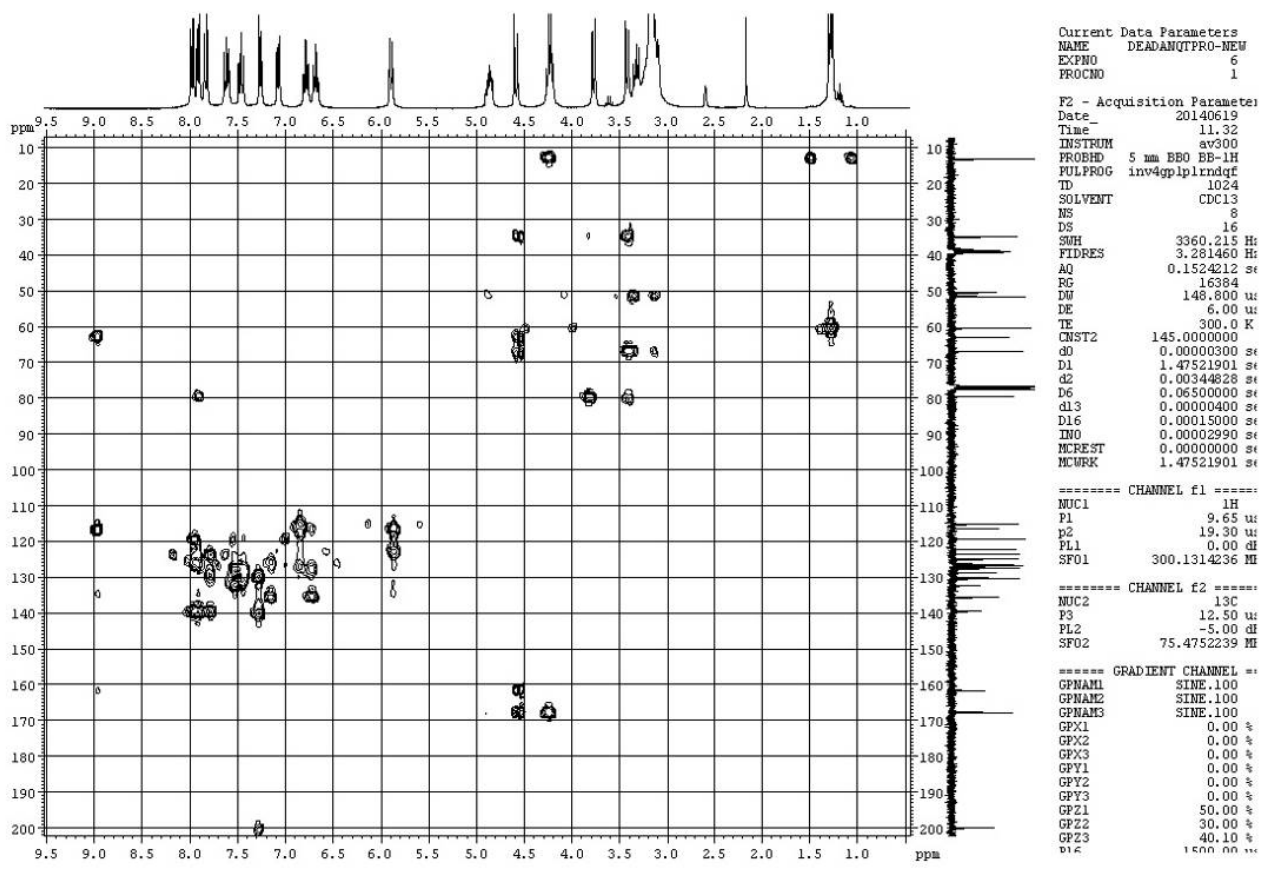

Figure 20. HMBC spectrum of $\mathbf{1 2 b}$ 


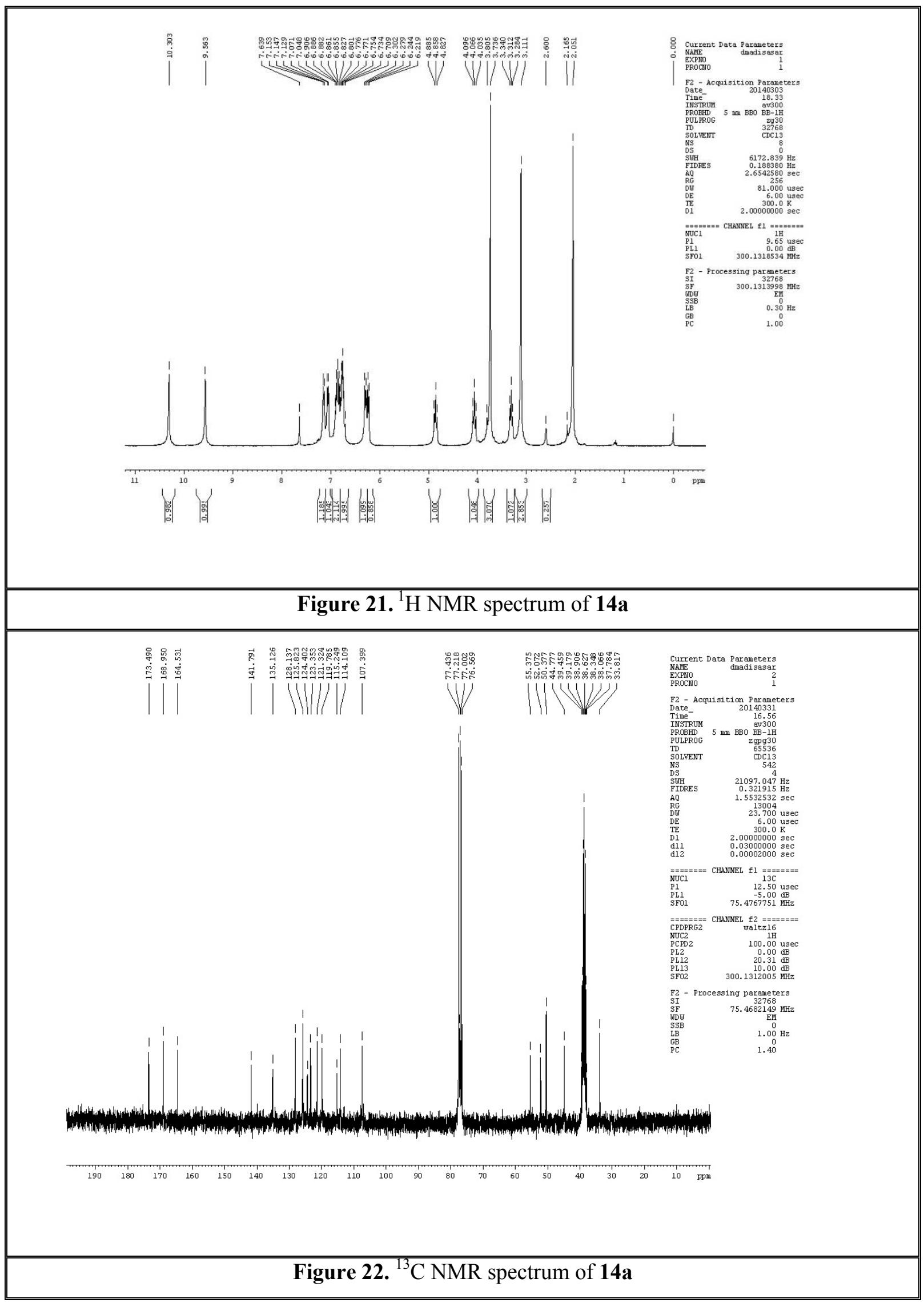




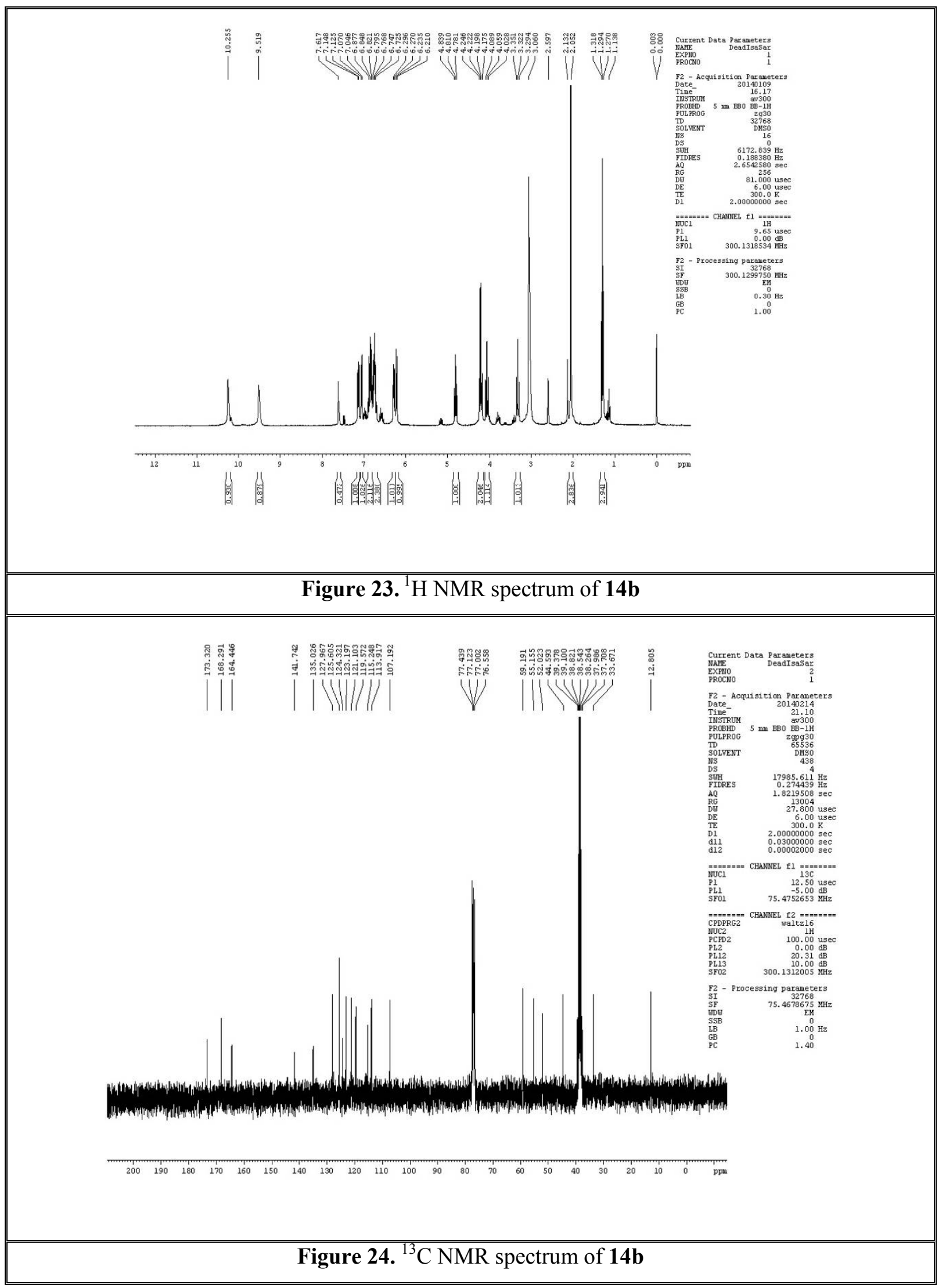




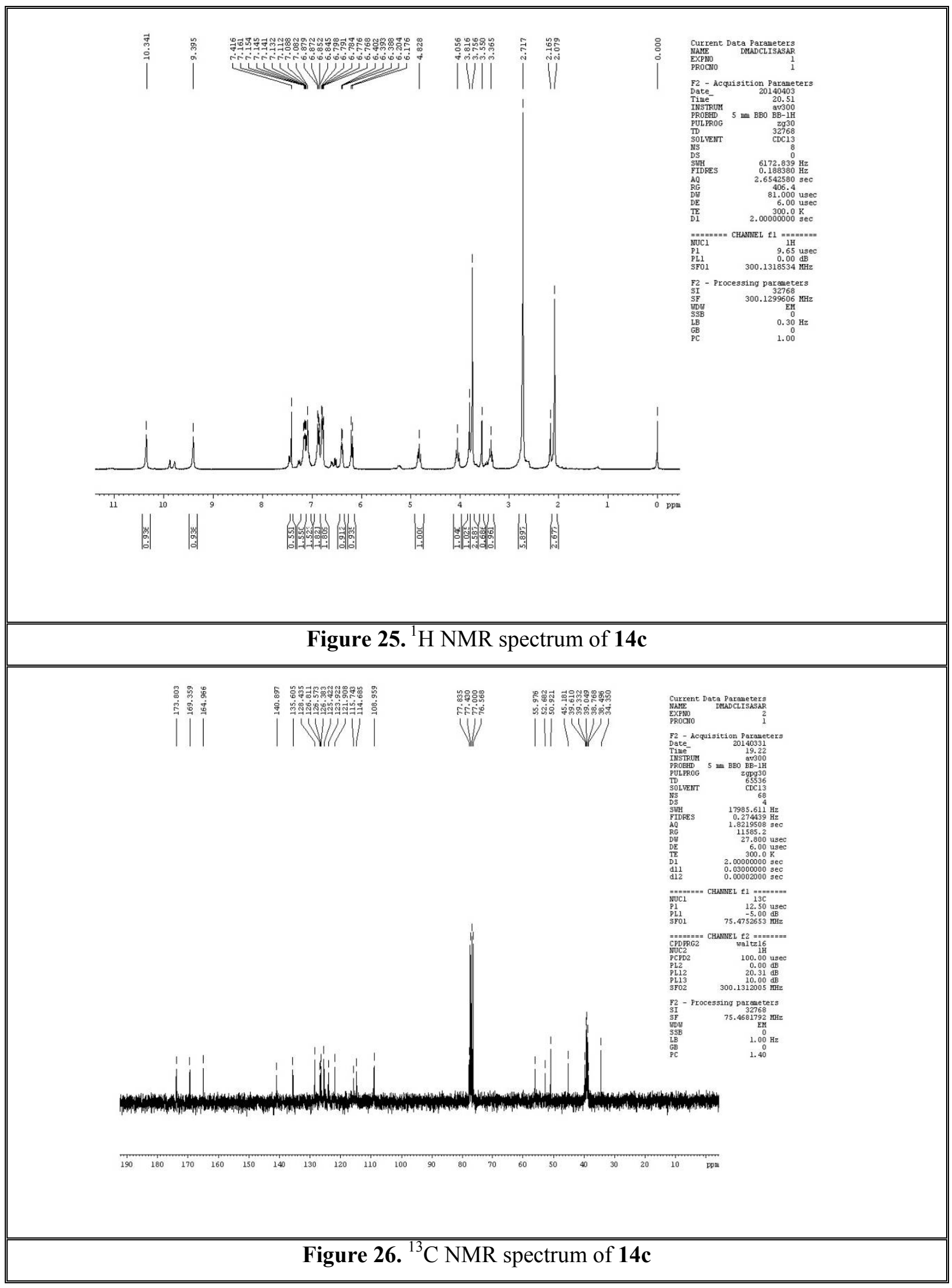




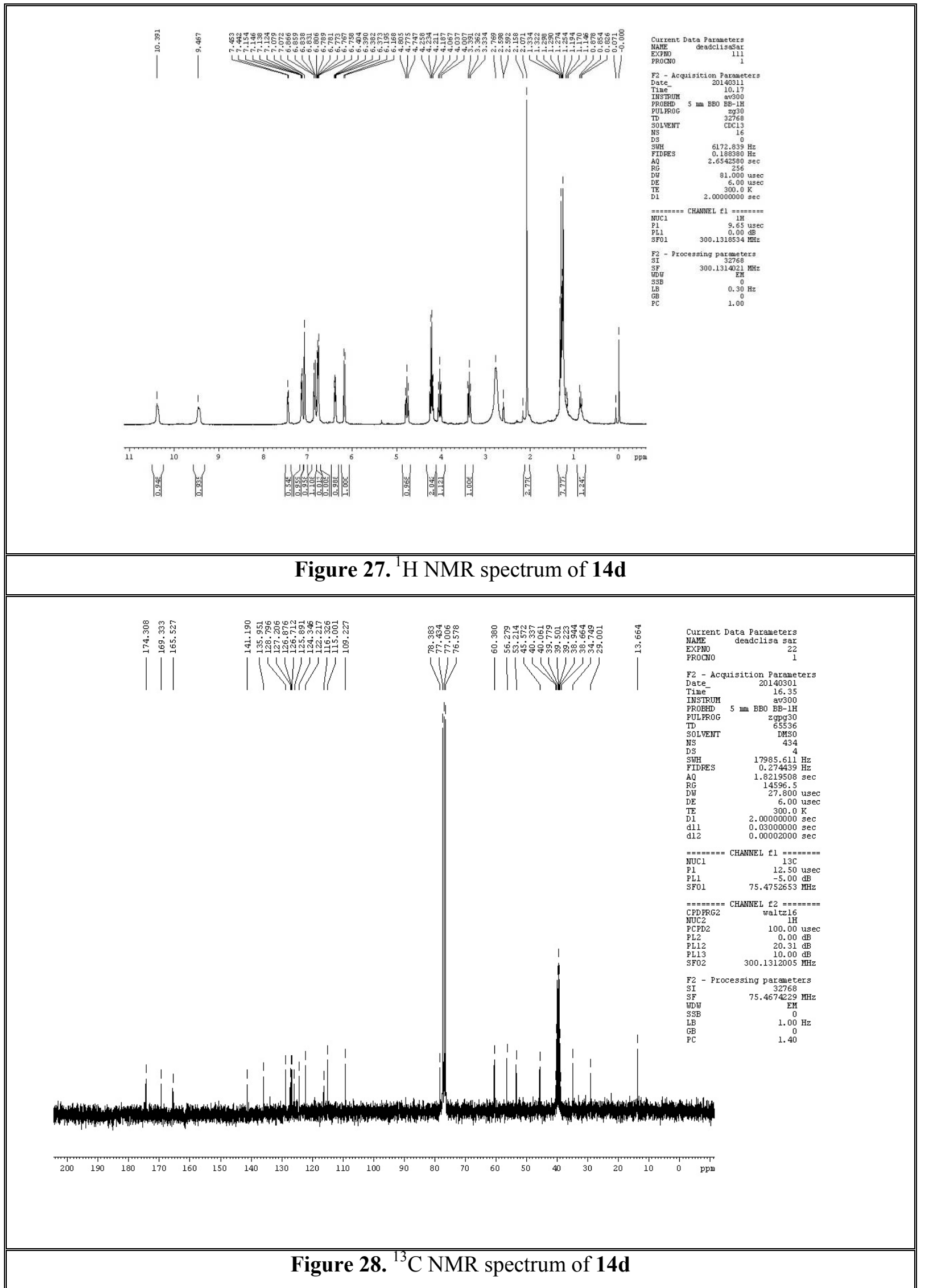




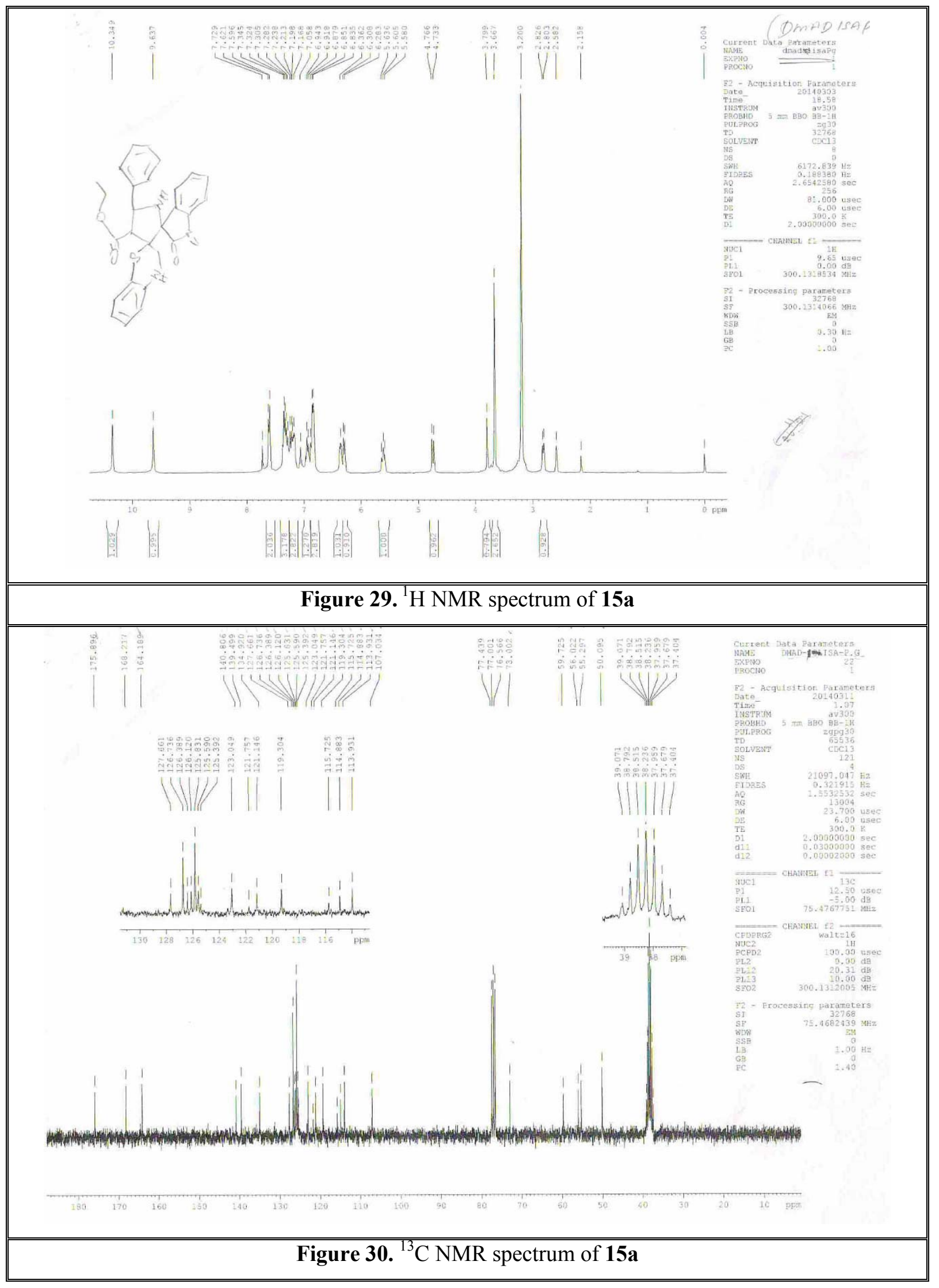




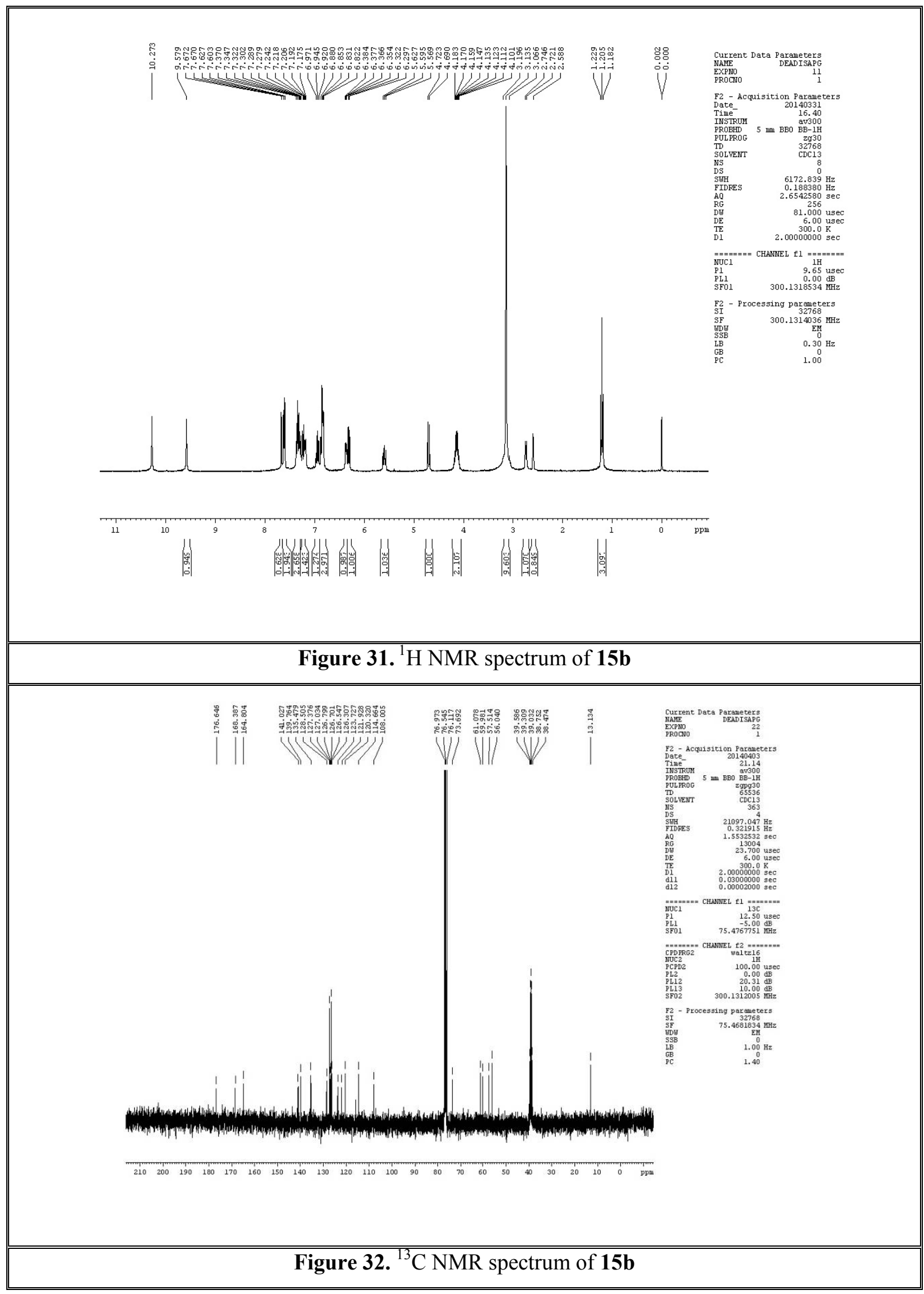




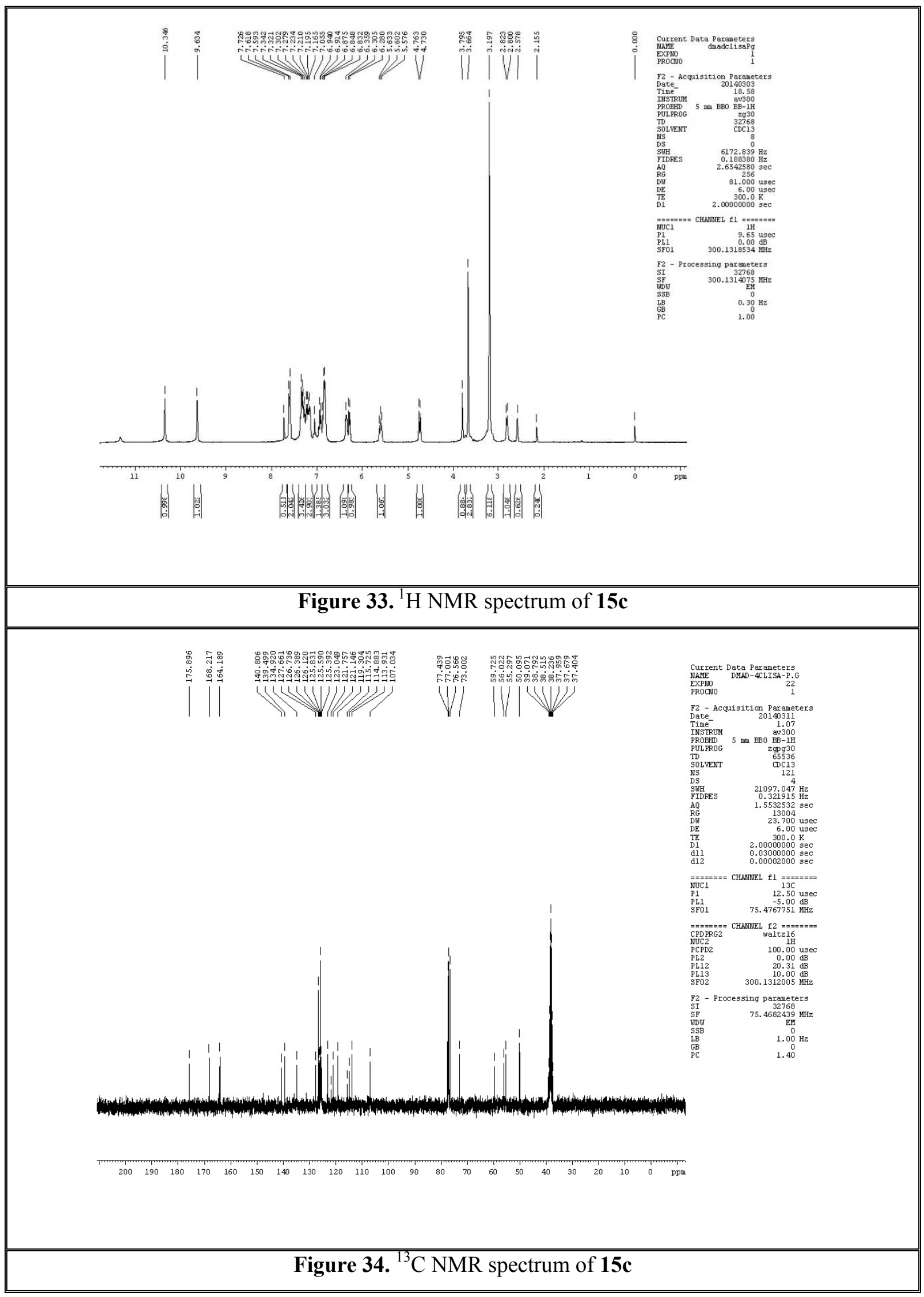




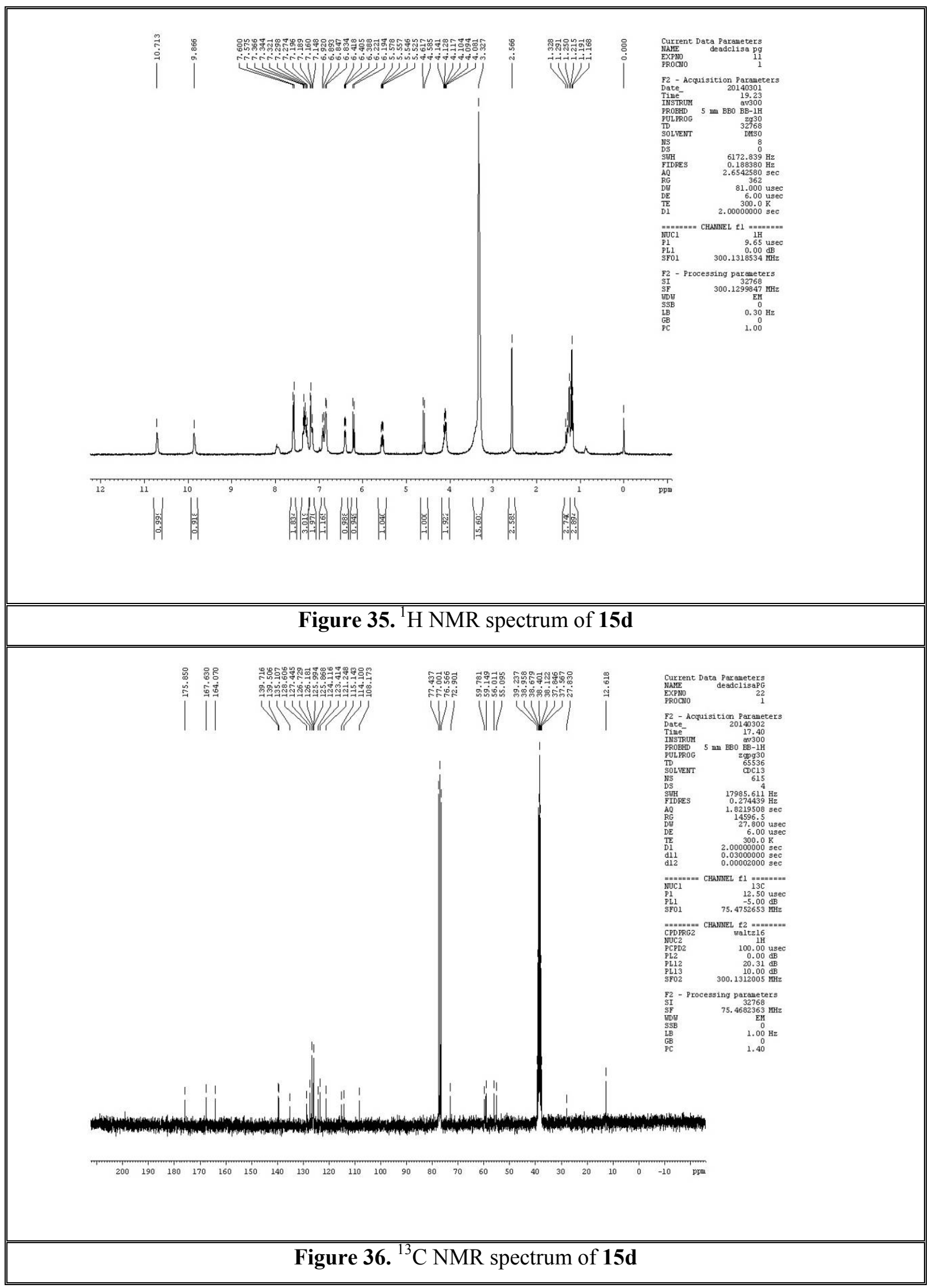




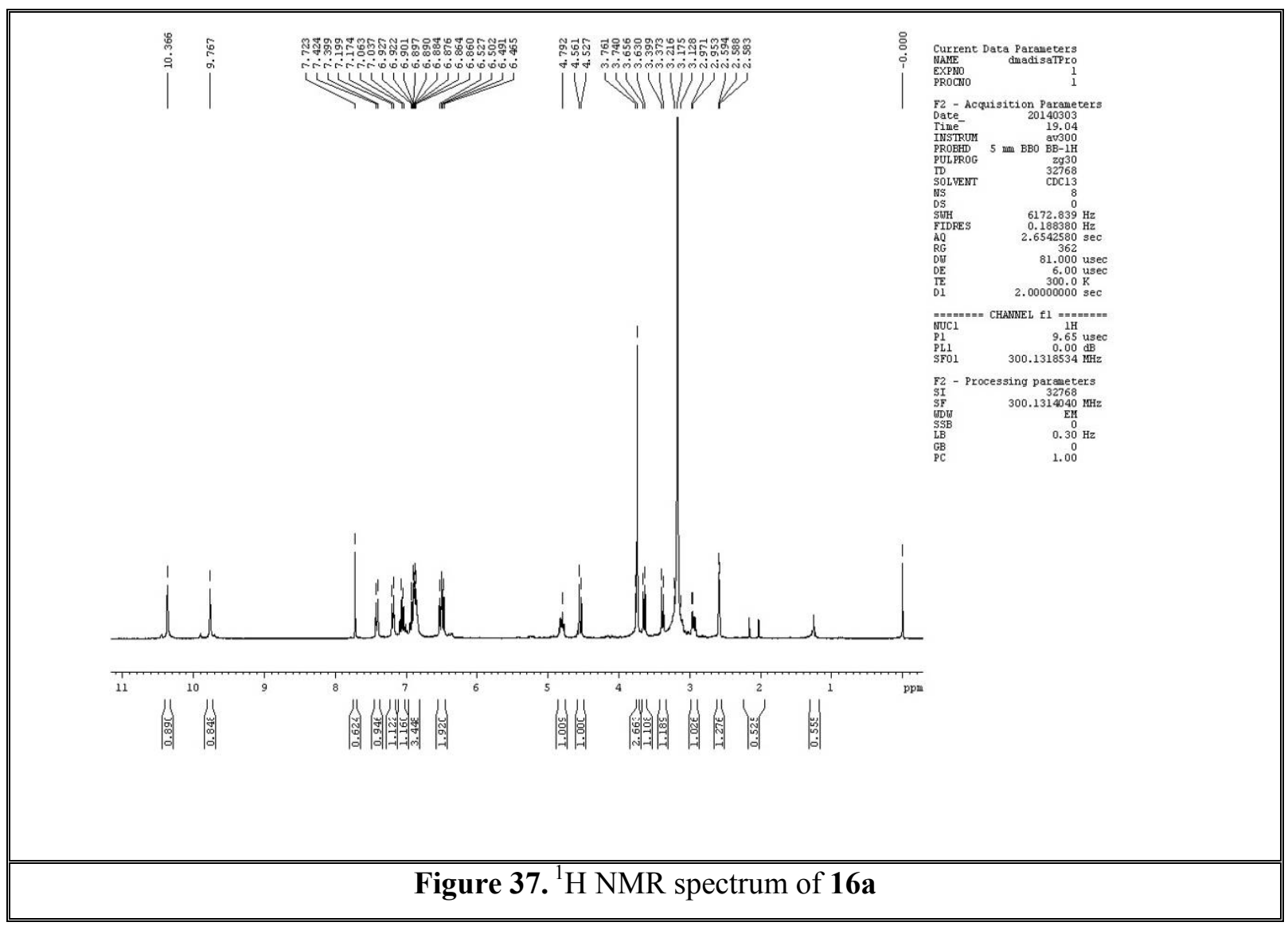




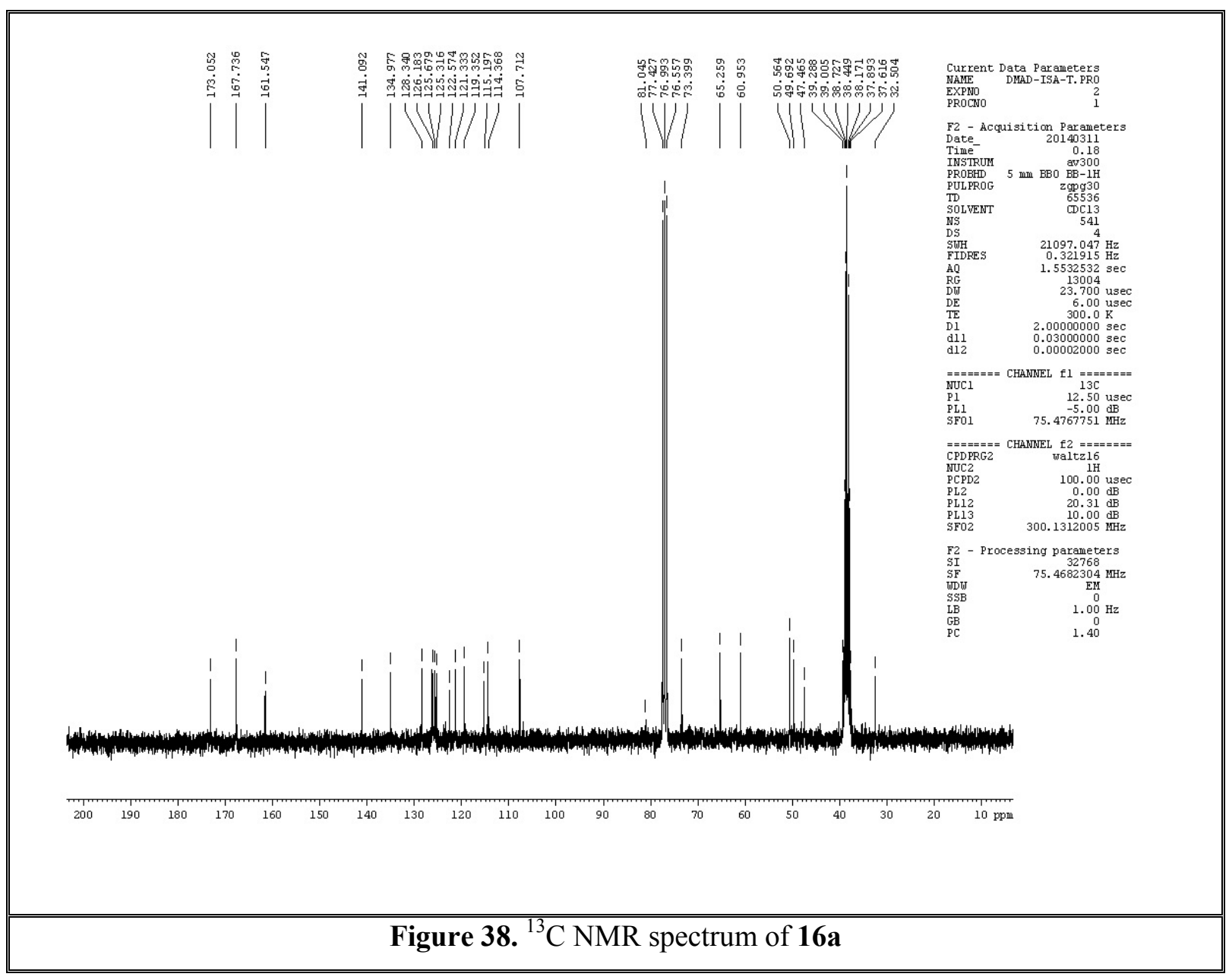




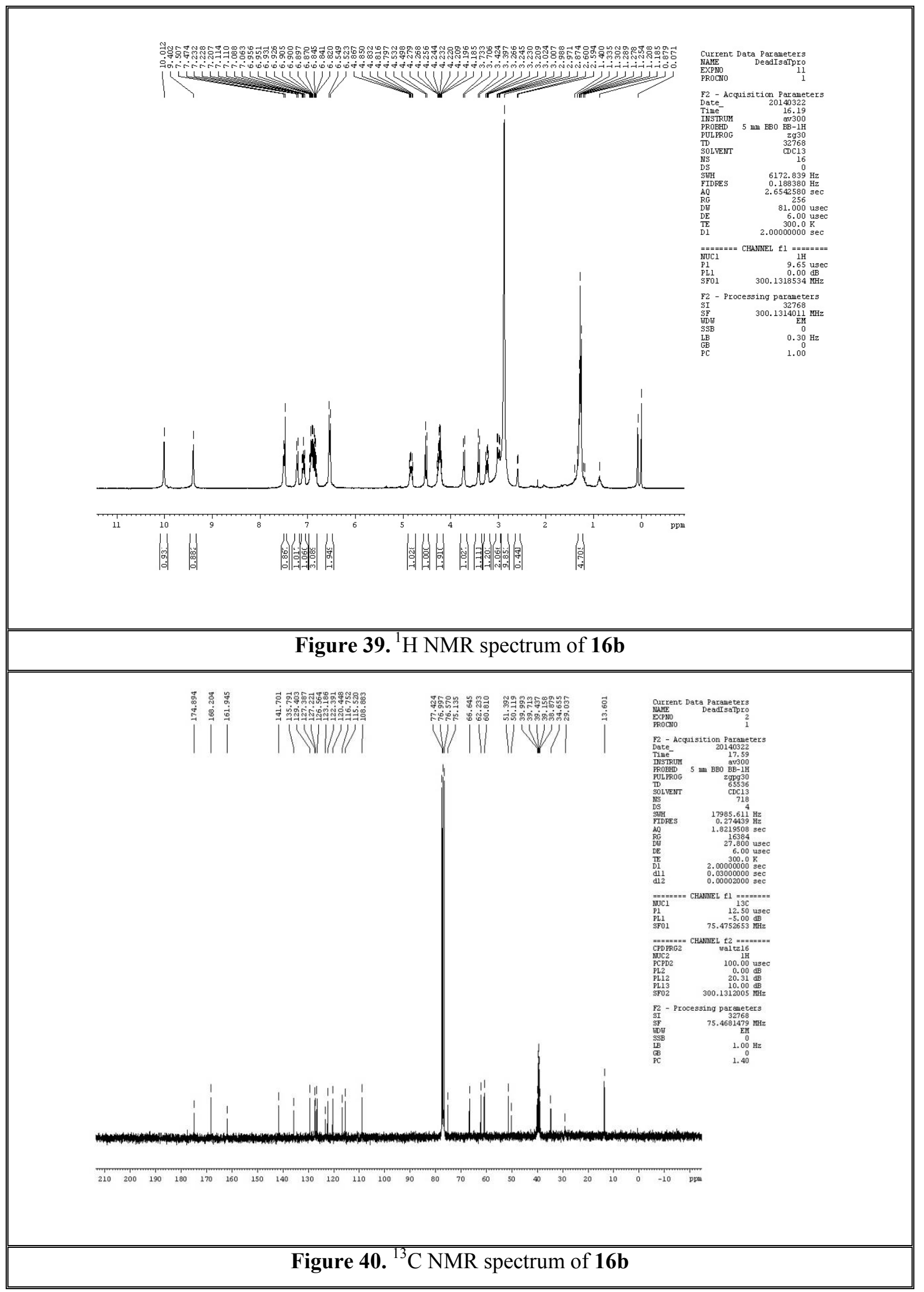




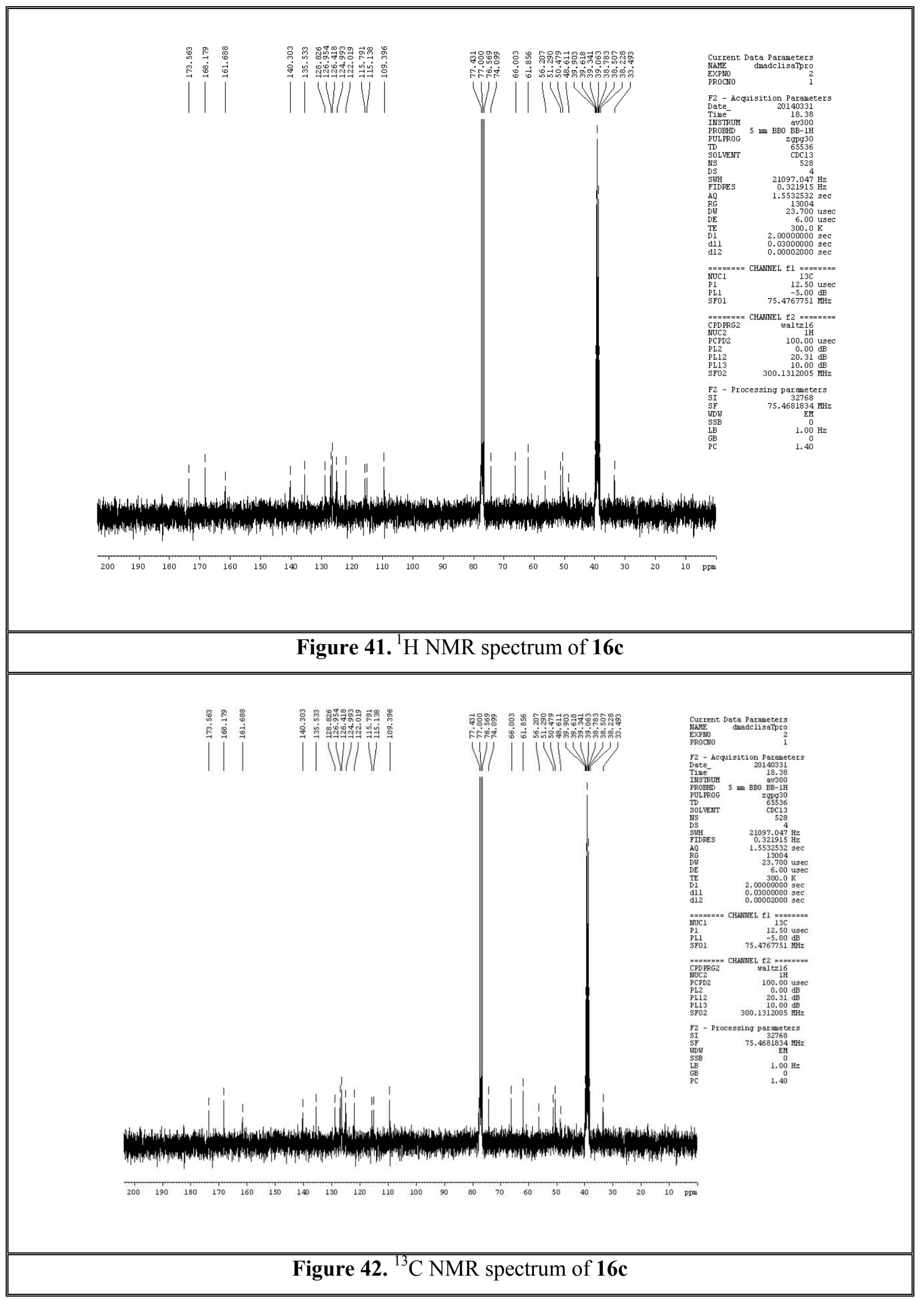




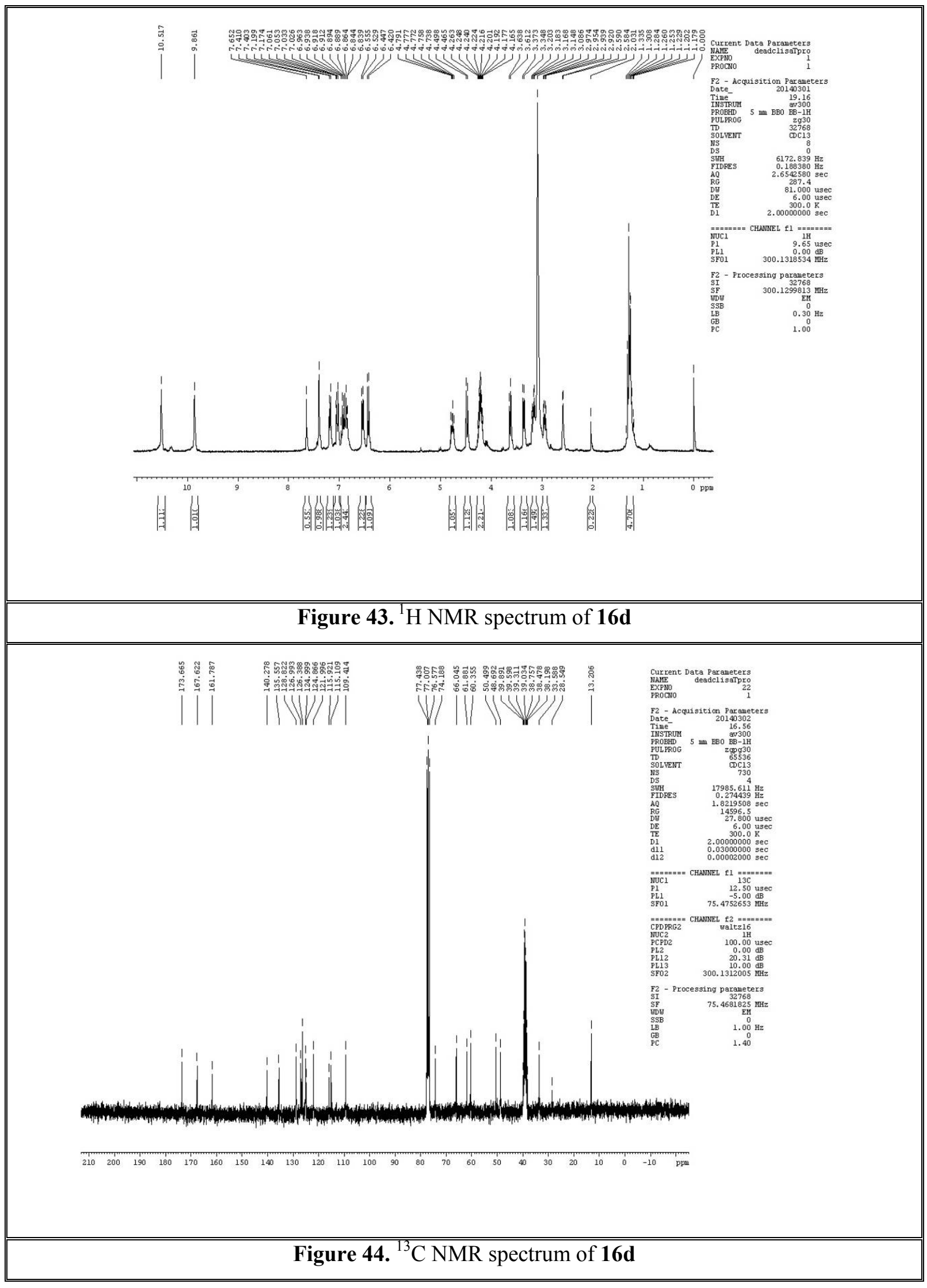

